\title{
LAGRANGIAN AND HAMILTONIAN FEYNMAN FORMULAE FOR SOME FELLER SEMIGROUPS AND THEIR PERTURBATIONS
}

\author{
YANA A. BUTKO, RENÉ L. SCHILLING, AND OLEG G. SMOLYANOV
}

\begin{abstract}
A Feynman formula is a representation of a solution of an initial (or initial-boundary) value problem for an evolution equation (or, equivalently, a representation of the semigroup resolving the problem) by a limit of $n$-fold iterated integrals of some elementary functions as $n \rightarrow \infty$. In this note we obtain some Feynman formulae for a class of semigroups associated with Feller processes. Finite dimensional integrals in the Feynman formulae give approximations for functional integrals in some Feynman-Kac formulae corresponding to the underlying processes. Hence, these Feynman formulae give an effective tool to calculate functional integrals with respect to probability measures generated by these Feller processes and, in particular, to obtain simulations of Feller processes.

KEYwords Feynman formulae; Feynman-Kac formulae; approximations of functional integrals, approximations of transition densities.
\end{abstract}

MSC 2010: 47D07, 47D08, 35C99, 60J35, 60J51, 60J60.

\section{Introduction}

In this note we consider a class of semigroups associated with Feller processes. Feller processes are continuous-time Markov processes, which generalize the class of stochastic processes with stationary and independent increments, or Lévy processes. Note that many diffusion processes belong to this class. Every Feller process $\left(\xi_{t}\right)_{t \geq 0}$ in $\mathbb{R}^{d}$ generates a strongly continuous positivity preserving contraction semigroup $\left(T_{t}\right)_{t \geq 0}$ on the space $C_{\infty}\left(\mathbb{R}^{d}\right)$ of continuous functions vanishing at infinity: $T_{t} f(q)=$ $\mathbb{E}^{q}\left[f\left(\xi_{t}\right)\right]$ for any $f \in C_{\infty}\left(\mathbb{R}^{d}\right)$. Due to Courrège it is known that (under a mild richness condition on the domain) the infinitesimal generator $A$ of a Feller semigroup is a pseudo-differential operator ( $\Psi \mathrm{DO}$, for short), i.e. an operator of the form

$A f(q)=-H(q, D) f(q)=-(2 \pi)^{-d} \int_{\mathbb{R}^{d}} \int_{\mathbb{R}^{d}} e^{i(q-x) \cdot p} H(q, p) f(x) d x d p, \quad f \in C_{c}^{\infty}\left(\mathbb{R}^{d}\right)$.

The symbol of the operator, the function $-H: \mathbb{R}^{d} \times \mathbb{R}^{d} \rightarrow \mathbb{C},(q, p) \mapsto-H(q, p)$, is for fixed $q$, given in terms of a Lévy-Khintchine representation

$$
H(q, p)=a(q)+i \ell(q) \cdot p+p \cdot Q(q) p+\int_{y \neq 0}\left(1-e^{i p \cdot y}+\frac{i p \cdot y}{1+|y|^{2}}\right) N(q, d y),
$$

where, for each fixed $q, \ell(q) \in \mathbb{R}^{d}, Q(q)$ is a positive semidefinite symmetric matrix and $N(q, d y)$ is a measure kernel on $\mathbb{R}^{d} \backslash\{0\}$ such that $\int_{y \neq 0} \frac{|y|^{2}}{1+|y|^{2}} N(q, d y)<\infty$. Note that these negative definite symbols do not belong to any of the classical symbol classes of $\Psi$ DOs; consequently we do not have a Hörmander or Maslov symbolic calculus at our disposal.

In a similar way, each operator $T_{t}$ can be represented as a pseudo-differential operator $\lambda_{t}(\cdot, D)$ with the symbol $\lambda_{t}(q, p)=\mathbb{E}^{q}\left[e^{i\left(\xi_{t}-q\right) \cdot p}\right]$. It is known that $-H(q, p)=$ 
$\lim _{t \rightarrow 0} \frac{\lambda_{t}(q, p)-1}{t}$, see e.g. [21, 30, 31. If $\left(\xi_{t}\right)_{t \geq 0}$ is a Lévy process, we have $H(q, p)=$ $H(p)$ and $\lambda_{t}(q, p)=e^{-t H(p)}$ - this is due to the fact that the generator is an operator with constant "coefficients" (i.e. it is independent of the state space variable $q)$. In the case where we have variable "coefficients", there is no such straightforward connection between the symbols of the semigroup and the generator and this gives rise to several interesting problems: which negative definite symbols $H(q, p)$ lead to Feller processes and, if so, how can we represent or approximate the symbol $\lambda_{t}(q, p)$. The existence problem has been discussed at length in a series of papers, see [22, 4, 24] and the literature given there, and we would like now to investigate the problem how to represent the semigroup resp. its symbol if the (symbol of the) generator is known.

Consider an evolution equation $\frac{\partial f}{\partial t}(t, q)=-H(q, D) f(t, q)$, where $-H(\cdot, D)$ is a generator of some Feller process $\left(\xi_{t}\right)_{t \geq 0}$. Following the terminology of mathematical physics, we call $H(\cdot, D)$ the Hamiltonian of the physical system, which is described by the above evolution equation. The solution of the Cauchy problem for this equation with initial data $f(0, q)=f_{0}(q)$ can be obtained by the Feynman-Kac formula $f(t, q) \equiv\left(T_{t} f_{0}\right)(q)=\mathbb{E}^{q}\left[f_{0}\left(\xi_{t}\right)\right]$. Here the expectation $\mathbb{E}^{q}\left[f_{0}\left(\xi_{t}\right)\right]$ is a functional integral (path integral) over the set of paths of the process $\left(\xi_{t}\right)_{t \geq 0}$ with respect to the measure generated by this process. If $\left(\xi_{t}\right)_{t \geq 0}$ is a diffusion process, then $\mathbb{E}^{q}\left[f_{0}\left(\xi_{t}\right)\right]=\int_{C\left([0, t], \mathbb{R}^{d}\right)} f_{0}\left(\xi_{t}\right) \mu(d \xi)$, where $\mu$ is a Gaussian measure, corresponding to this process; in particular, the Wiener measure corresponds to the process of Brownian motion.

The heuristic notion of a path integral has been introduced by R. Feynman, see [16], 17, to obtain the solution of the Schrödinger equation with a potential. Feynman has defined this integral as a limit of some finite dimensional integrals; actually, these integrals range over Cartesian powers of the configuration (or phase) space of the system, described by the Schrödinger equation. In modern terminology this kind of path integrals are called Feynman path integrals with respect to a Feynman pseudomeasure on the set of paths in the configuration (or phase) space.

The classical Feynman-Kac formula, representing the solution of the Cauchy problem for the heat equation by a functional integral with respect to the Wiener measure can also be obtained applying Feynman's construction. Here the functional integral is a limit of $n$-fold iterated integrals containing Gaussian exponents which are transition densities of a Brownian motion. This construction can be extended to a large class of Markov processes. However, in most cases the transition densities of Feller processes cannot be expressed by elementary functions and, hence, in order to compute functional integrals in Feynman-Kac formulae we need to approximate them. This gives rise to Feynman formulae.

A Feynman formula is a representation of the solution of an initial (or initialboundary) value problem for an evolution equation (or, equivalently, a representation of the semigroup resolving the problem) as a limit of $n$-fold iterated integrals of some elementary functions, when $n \rightarrow \infty$. Obviously, the iterated integrals in a Feynman formula for some problem give approximations for a functional integral in the Feynman-Kac formula representing the solution of the problem. These approximations can be used for direct calculations and simulations.

The notion of a Feynman formula has been introduced in 33 and the method to obtain Feynman formulae for evolutionary equations has been developed in a series of papers [33-37]. Recently, this method has been successfully applied to obtain Feynman formulae for different classes of problems for evolutionary equations on different geometric structures, see, e.g. [5]- 7], 26], 27], 32] and also to construct some surface measures on infinite dimensional manifolds (see 34-38]). This method is based on Chernoff's theorem (see [12] and [33 for the version used 
here), which is a generalization of the well-known Trotter formula. Trotter's formula has been used to justify Feynman's heuristic result for Schrödinger equations with a potential, e.g. 25, and to prove the classical Feynman-Kac formula mentioned earlier.

By Chernoff's theorem a strongly continuous semigroup $\left(T_{t}\right)_{t \geq 0}$ on a Banach space can be represented as a strong limit: $T_{t}=\lim _{n \rightarrow \infty}[F(t / n)]^{n}$ where $F(t)$ is an operator-valued function satisfying certain conditions (see Theorem 2.4 for details). This equality is called a Feynman formula for the semigroup $\left(T_{t}\right)_{t \geq 0}$. We call this Feynman formula a Lagrangian Feynman formula, if the $F(t), t>0$, are integral operators with elementary kernels; if the $F(t)$ are $\Psi$ DOs, we speak of Hamiltonian Feynman formulae. In particular, we obtain a Hamiltonian Feynman formula for a semigroup $T_{t} \equiv e^{-t H(\cdot, D)}$ generated by a $\Psi \mathrm{DO}-H(\cdot, D)$ with the symbol $-H(q, p)$ if

$$
e^{-t H(\cdot, D)}=\lim _{n \rightarrow \infty}\left[e^{-\frac{t}{n} H}(\cdot, D)\right]^{n},
$$

where $e^{-\frac{t}{n} H}(\cdot, D)$ is the $\Psi$ DO with the symbol $e^{-\frac{t}{n} H(q, p)}$. Note that, in general $e^{-\frac{t}{n} H}(\cdot, D)$ is not a semigroup and that $\lambda_{t}(q, p) \neq e^{-t H(q, p)}$.

Our terminology is inspired by the fact that a Lagrangian Feynman formula gives approximations to a functional integral over a set of paths in the configuration space of a system (whose evolution is described by the semigroup $\left(T_{t}\right)_{t \geq 0}$ ), while a Hamiltonian Feynman formula corresponds to a functional (Hamiltonian Feynman path) integral over a set of paths in the phase space of some system (cf. [3] ). The corresponding Hamiltonian Feynman formula gives rise to a Hamiltonian Feynman path integral also in the case $T_{t}=e^{i t H(q, D)}$ (see 33]).

In this note we prove some Hamiltonian and Lagrangian Feynman formulae for semigroups associated with Feller processes and for perturbations of such semigroups. Several results of the paper have been announced in [9]. The paper is organized as follows. Section 2 contains notation and some preliminaries; in particular, Chernoff's theorem is formulated and the notion of Chernoff equivalence is introduced. In Section 3 we prove a Hamiltonian Feynman formula for a class of semigroups associated with Feller processes. In Section 4 we obtain a Lagrangian Feynman formula for a multiplicative perturbation of a Feller semigroup by a function $a(\cdot)$ which is continuous, positive, bounded and bounded away from zero. Note, that analogous Lagrangian Feynman formulas have been proved for some diffusion processes in 8 and have been presented for the Cauchy process in 9]. In Section 5 we consider gradient and bounded Schrödinger perturbations of Feller semigroups and obtain some Hamiltonian and Lagrangian Feynman formulae for them.

\section{Notations and preliminaries}

Let $C_{c}^{\infty}\left(\mathbb{R}^{d}\right)$ be a set of infinitely differentiable functions on $\mathbb{R}^{d}$ with compact support and $S\left(\mathbb{R}^{d}\right)$ be the Schwartz space of rapidly decreasing functions. Let us also consider a space $C_{\infty}\left(\mathbb{R}^{d}\right)$ of all continuous functions vanishing at infinity. It is a Banach space with the norm $\|f\|_{\infty}=\sup _{x \in \mathbb{R}^{d}}|f(x)|$. Write for the norm in $C_{\infty}^{k}\left(\mathbb{R}^{d}\right)$, the space of $k$ times continuously differentiable functions which vanish (with all their derivatives) at infinity,

$$
\|u\|_{(k)}:=\sum_{|\alpha| \leqslant k}\left\|\partial^{\alpha} u\right\|_{\infty}
$$

where $\alpha \in \mathbb{N}_{0}^{n}, \partial^{\alpha}=\partial^{|\alpha|} / \partial x_{1}^{\alpha_{1}} \ldots \partial x_{n}^{\alpha_{n}}$, and $|\alpha|=\alpha_{1}+\ldots+\alpha_{n}$.

We use the following notations for the Fourier transform and its inverse:

$$
\widehat{f}(p)=(2 \pi)^{-d / 2} \int_{\mathbb{R}^{d}} e^{-i p \cdot q} f(q) d q \quad \text { and } \quad \mathcal{F}^{-1}[f](q)=(2 \pi)^{-d / 2} \int_{\mathbb{R}^{d}} e^{i p \cdot q} f(p) d p .
$$


2.1. Negative definite functions. Negative definite functions have been introduced by I.J. Schönberg in connection with isometric embeddings of metric spaces into a Hilbert space. His original definition is the following.

Definition 2.1. A function $\psi: \mathbb{R}^{d} \rightarrow \mathbb{C}$ is called negative definite if for any $m \in \mathbb{N}$ and all $p_{1}, \ldots, p_{m} \in \mathbb{R}^{d}$ the $m \times m$ matrix $\left(\psi\left(p_{j}\right)+\overline{\psi\left(p_{k}\right)}-\psi\left(p_{j}-p_{k}\right)\right)_{j, k=1, \ldots, m}$ is positive hermitian, i.e., if for all $\lambda_{1}, \ldots, \lambda_{m} \in \mathbb{C}$

$$
\sum_{j, k=1}^{m}\left(\psi\left(p_{j}\right)+\overline{\psi\left(p_{k}\right)}-\psi\left(p_{j}-p_{k}\right)\right) \lambda_{j} \overline{\lambda_{k}} \geqslant 0 .
$$

A negative definite function is NOT the negative of a positive definite function. Recall that a function $u: \mathbb{R}^{d} \rightarrow \mathbb{C}$ is called positive definite if for any choice of $k \in \mathbb{N}$ and vectors $p_{1}, \ldots, p_{k} \in \mathbb{R}^{d}$ the matrix $\left(u\left(p_{i}-p_{j}\right)\right)_{i, j=1, \ldots, k}$ is positive Hermitian, i.e. for all $\lambda_{1}, \ldots, \lambda_{k} \in \mathbb{C}$ we have $\sum_{i, j=1}^{k} u\left(p_{i}-p_{j}\right) \lambda_{i} \bar{\lambda}_{j} \geq 0$.

Corollary 2.1. If $u: \mathbb{R}^{d} \rightarrow \mathbb{C}$ is a positive definite function, then the function $[p \mapsto u(0)-u(p)]$ is negative definite.

The deeper connection between positive definite and negative definite functions can be seen from the following Theorem 2.1 which also justifies the definition of continuous negative definite functions through the Lévy-Khintchine formula:

Definition 2.2. A function $\psi: \mathbb{R}^{d} \rightarrow \mathbb{C}$ is called a continuous negative definite function if $\psi$ is given by the Lévy-Khintchine formula

$$
\psi(p)=a+i \ell \cdot p+p \cdot Q p+\int_{y \neq 0}\left(1-e^{i y \cdot p}+\frac{i y \cdot p}{1+|y|^{2}}\right) N(d y) .
$$

The tuple $(a, \ell, Q, N)$ consisting of $a \in \mathbb{R}^{+}, \ell \in \mathbb{R}^{d}$, a positive semidefinite matrix $Q \in \mathbb{R}^{d \times d}$ and a Radon measure $N$ on $\mathbb{R}^{d} \backslash\{0\}$ with $\int_{y \neq 0}|y|^{2}\left(1+|y|^{2}\right)^{-1} N(d y)<\infty$ is called Lévy characteristics (of $\psi$ ). The measure $N$ is often called Lévy measure.

Obviously, the Lévy characteristics are uniquely determined by $\psi$-and vice versa.

Theorem 2.1. For $\psi: \mathbb{R}^{d} \rightarrow \mathbb{C}$ the following properties are equivalent:

(a) $\psi$ is continuous and negative definite in the sense of Definition [2.1;

(b) $\psi$ is given by the Lévy-Khintchine formula (1);

(c) $\psi(0) \geqslant 0$ and $e^{-t \psi}$ is for every $t>0$ continuous and positive definite.

A proof of Theorem 2.1 can be found, e.g. in the monographs by Jacob 22 or by Berg and Forst [1] (II. $\$ 7)$. All continuous positive definite functions are characterized by Bochner's Theorem.

Theorem 2.2 (Bochner). A function $\phi: \mathbb{R}^{d} \rightarrow \mathbb{C}$ is continuous and positive definite if, and only if, it is the Fourier transform of a bounded Radon measure $\mu \in \mathcal{M}_{b}^{+}\left(\mathbb{R}^{d}\right)$, i.e.,

$$
\phi(p)=\widehat{\mu}(p):=(2 \pi)^{-d / 2} \int_{\mathbb{R}^{d}} e^{-i p \cdot q} \mu(d q) .
$$

From Definition 2.1 it is not hard to see that a negative definite function has positive real part $\operatorname{Re} \psi \geqslant 0$, satisfies $\overline{\psi(p)}=\psi(-p)$ and that $\sqrt{|\psi(\cdot)|}$ is subadditive, i.e.,

$$
\sqrt{\left|\psi\left(p_{1}+p_{2}\right)\right|} \leqslant \sqrt{\left|\psi\left(p_{1}\right)\right|}+\sqrt{\left|\psi\left(p_{2}\right)\right|}, \quad p_{1}, p_{2} \in \mathbb{R}^{d}
$$


If $\psi$ is continuous, repeated applications of the subadditivity estimate yield the following growth bound

$$
|\psi(p)| \leqslant 2 \sup _{|\eta| \leqslant 1}|\psi(\eta)|\left(1+|p|^{2}\right), \quad p \in \mathbb{R}^{d} .
$$

2.2. Feller and Lévy semigroups and their generators. A Feller process $\left(X_{t}\right)_{t \geqslant 0}$ with a state space $\mathbb{R}^{d}$ is a strong Markov process whose associated operator semigroup $\left(T_{t}\right)_{t \geqslant 0}$,

$$
T_{t} u(x)=\mathbb{E}^{x}\left[u\left(X_{t}\right)\right], \quad u \in C_{\infty}\left(\mathbb{R}^{d}\right), t \geqslant 0, x \in \mathbb{R}^{d},
$$

enjoys the Feller property, i.e., it is a strongly continuous positivity preserving contraction semigroup on the space $C_{\infty}\left(\mathbb{R}^{d}\right)$. The semigroup $\left(T_{t}\right)_{t \geqslant 0}$ is said to be a Feller semigroup.

The (infinitesimal) generator $(A, D(A))$ of the semigroup or the process is given by the strong limit

$$
A u:=\lim _{t \rightarrow 0} \frac{T_{t} u-u}{t}
$$

on the set $D(A) \subset C_{\infty}\left(\mathbb{R}^{d}\right)$ of those $u \in C_{\infty}\left(\mathbb{R}^{d}\right)$ for which the above limit exists w.r.t. the sup-norm. We will call $(A, D(A))$ a Feller generator for short.

Before we proceed with general Feller semigroups it is instructive to have a brief look at Lévy processes (and convolution semigroups) which are a particular subclass of Feller processes. Our standard reference for Lévy processes is the monograph by K. Sato [29]. A Lévy process $\left(Y_{t}\right)_{t \geqslant 0}$ is a stochastically continuous random process with stationary and independent increments. The Fourier transform of a Lévy process has a particularly simple structure,

$$
\mathbb{E}^{x}\left[e^{i p \cdot\left(Y_{t}-x\right)}\right]=\mathbb{E}^{0}\left[e^{i p \cdot Y_{t}}\right]=e^{-t \psi(p)},
$$

where $\psi: \mathbb{R}^{d} \rightarrow \mathbb{C}$ is the characteristic exponent which is a continuous negative definite function, i.e. $\psi$ is given by the Lévy-Khintchine formula (11). Since $\left(Y_{t}\right)_{t \geqslant 0}$ is a Markov process both (3) and (1) characterize the finite dimensional distributions of $\left(Y_{t}\right)_{t \geqslant 0}$ and, hence, the process itself.

A Lévy process is spatially homogeneous. Therefore, the associated semigroup is of convolution type,

$$
S_{t} u(x)=\mathbb{E}^{x}\left[u\left(Y_{t}\right)\right]=\mathbb{E}^{0}\left[u\left(Y_{t}+x\right)\right]=\int_{\mathbb{R}^{d}} u(x+y) \mathbb{P}^{0}\left(Y_{t} \in d y\right)=u * \tilde{\mu}_{t}(d y),
$$

$\tilde{\mu}_{t}(d y)=\mathbb{P}^{0}\left(Y_{t} \in-d y\right)$, and a short direct calculation shows that $\left(S_{t}\right)_{t \geqslant 0}$ is indeed a Feller semigroup with infinitesimal generator

$$
B u(x)=-\psi(D) u(x):=-(2 \pi)^{-n / 2} \int_{\mathbb{R}^{d}} \psi(p) \widehat{u}(p) e^{i x \cdot p} d p, \quad u \in C_{c}^{\infty}\left(\mathbb{R}^{d}\right) .
$$

One can use the estimate (2) to show that integrals in (44) are convergent.

The operator $\psi(D)$ is a first example of a so-called pseudo differential operator with the symbol $\psi(p)$. Since $\psi$ does not depend on $x$ the operator has constant "coefficients". Notice that the symbol $\psi$ is just the characteristic exponent of the process $\left(Y_{t}\right)_{t \geqslant 0}$. This shows that

every Lévy process is generated by a pseudo differential operator $-\psi(D)$ with the symbol $-\psi(p)$ where $\psi$ is the characteristic exponent of the process. Conversely, every pseudo differential operator $-\psi(D)$ with the symbol $-\psi(p)$, where $\psi$ is a continuous negative definite function, i.e., given by the Lévy-Khintchine formula (1), is the generator of a Lévy process. 
Let us return to the general situation. It is not hard to see (cf. 15,p. 165, Theorem $2.2(\mathrm{~b}))$ that Feller generators satisfy the so-called positive maximum principle

$(\mathrm{PMP}) \quad$ if $\quad u \in D(A), \quad \sup _{x \in \mathbb{R}^{d}} u(x)=u\left(x_{0}\right) \geqslant 0 \quad$ then $\quad A u\left(x_{0}\right) \leqslant 0$.

Extending earlier work of W. von Waldenfels [39, 40] $\mathrm{Ph}$. Courrège showed in 13, see also [2], the following structure result for operators satisfying the positive maximum principle. We formulate his theorem only for Feller generators.

Theorem 2.3 (Courrège). Let $(A, D(A))$ be a Feller generator such that $C_{c}^{\infty}\left(\mathbb{R}^{d}\right) \subset$ $D(A)$. Then $\left.A\right|_{C_{c}^{\infty}\left(\mathbb{R}^{d}\right)}$ is a pseudo differential operator,

$$
A u(q)=-H(q, D) u(q)=-(2 \pi)^{-n / 2} \int_{\mathbb{R}^{d}} H(q, p) \widehat{u}(p) e^{i p \cdot q} d p, \quad u \in C_{c}^{\infty}\left(\mathbb{R}^{d}\right),
$$

with the symbol $H: \mathbb{R}^{d} \times \mathbb{R}^{d} \rightarrow \mathbb{C}$ which is measurable, locally bounded in both variables $(q, p)$, and satisfies for fixed $q$ the following Lévy-Khintchine representation

(6) $H(q, p)=a(q)+i \ell(q) \cdot p+p \cdot Q(q) p+\int_{y \neq 0}\left(1-e^{i y \cdot p}+\frac{i y \cdot p}{1+|y|^{2}}\right) N(q, d y)$,

where $(a(q), \ell(q), Q(q), N(q, \cdot))$ is for each $q \in \mathbb{R}^{d}$ the Lévy characteristics of $-H(q, \cdot)$.

Observe that (6) automatically implies the continuity of $p \mapsto H(q, p)$ for each $q \in \mathbb{R}^{d}$.

Let $H(q, D)$ be a pseudo differential operator with the symbol $H(q, p)$ as in Theorem 2.3. Since $H(q, p)$ is represented by the Lévy-Khintchine type formula (6) we can use Fourier inversion in (5) and find that the integro-differential operator

$$
\begin{aligned}
A \varphi(q)=- & a(q) \varphi(q)+\ell(q) \cdot \nabla \varphi(q)+\sum_{j, k=1}^{d} Q^{j k}(x) \partial_{j} \partial_{k} \varphi(q) \\
& +\int_{y \neq 0}\left(\varphi(q+y)-\varphi(q)-\frac{y \cdot \nabla \varphi(q)}{1+|y|^{2}}\right) N(q, d y)
\end{aligned}
$$

extends $\left(-H(\cdot, D), C_{c}^{\infty}\left(\mathbb{R}^{d}\right)\right)$ to the set $C_{\infty}^{2}\left(\mathbb{R}^{n}\right)$. Note that the following Lemma 2.1 together with the integration properties of $N(q, d y)$,

$$
\int_{y \neq 0}|y|^{2} /\left(1+|y|^{2}\right) N(q, d y)<\infty
$$

ensure that the integral in (17) converges. From now on we will use the pseudo differential representation (5) and the integro-differential representation (7) simultaneously.

Lemma 2.1. For all $\varphi \in C_{b}^{2}\left(\mathbb{R}^{d}\right)$ we have

$$
\left|\varphi(q+y)-\varphi(q)-\frac{y \cdot \nabla \varphi(q)}{1+|y|^{2}}\right| \leqslant 2 \frac{|y|^{2}}{1+|y|^{2}}\|\varphi\|_{(2)} .
$$


Proof. By Taylor's formula we get for all $q, y \in \mathbb{R}^{d}$

$$
\begin{aligned}
\mid\left(1+|y|^{2}\right) & \left(\varphi(q+y)-\varphi(q)-\frac{y \cdot \nabla \varphi(q)}{1+|y|^{2}}\right) \mid \\
& \leqslant|\varphi(q+y)-\varphi(q)-y \cdot \nabla \varphi(q)|+|y|^{2}|\varphi(q+y)-\varphi(q)| \\
& \leqslant \frac{1}{2}\left|\sum_{j, k=1}^{d} y_{j} y_{k} \partial_{j} \partial_{k} \varphi\left(\xi_{q, y}\right)\right|+2|y|^{2}\|\varphi\|_{\infty} \\
& \leqslant 2|y|^{2}\left(\|\varphi\|_{\infty}+\sqrt{\left.\sum_{j, k=1}^{d}\left\|\partial_{j} \partial_{k} \varphi\right\|_{\infty}^{2}\right)}\right. \\
& \leqslant 2|y|^{2}\|\varphi\|_{(2)} .
\end{aligned}
$$

In the sequel we will need also the following Lemma.

Lemma 2.2. We have

$$
\frac{|y|^{2}}{1+|y|^{2}}=\int_{\mathbb{R}^{d}}(1-\cos (y \cdot p)) g(p) d p, \quad y \in \mathbb{R}^{d},
$$

where $g(p)=\frac{1}{2} \int_{0}^{\infty}(2 \pi \lambda)^{-d / 2} e^{-|p|^{2} / 2 \lambda} e^{-\lambda / 2} d \lambda$ is integrable and has absolute moments of arbitrary order.

Proof. The Tonelli-Fubini Theorem and a change of variables show for $k \in \mathbb{N}_{0}$

$$
\begin{aligned}
\int_{\mathbb{R}^{d}}|p|^{k} g(p) d p & =\frac{1}{2} \int_{0}^{\infty}(2 \pi \lambda)^{-d / 2} \int_{\mathbb{R}^{d}}|p|^{k} e^{-|p|^{2} / 2 \lambda} d p e^{-\lambda / 2} d \lambda \\
& =\frac{1}{2} \int_{0}^{\infty}(2 \pi \lambda)^{-d / 2} \int_{\mathbb{R}^{d}} \lambda^{k / 2}|\eta|^{k} e^{-|\eta|^{2} / 2} \lambda^{d / 2} d \eta e^{-\lambda / 2} d \lambda \\
& =\frac{1}{2}(2 \pi)^{-d / 2} \int_{\mathbb{R}^{d}}|\eta|^{k} e^{-|\eta|^{2} / 2} d \eta \int_{0}^{\infty} \lambda^{k / 2} e^{-\lambda / 2} d \lambda,
\end{aligned}
$$

i.e., $g$ has absolute moments of any order. Moreover, the elementary formula

$$
e^{-\lambda|y|^{2} / 2}=(2 \pi \lambda)^{-d / 2} \int_{\mathbb{R}^{n}} e^{-|p|^{2} / 2 \lambda} e^{i y \cdot p} d p
$$

and Fubini's Theorem yield

$$
\begin{aligned}
\frac{|y|^{2}}{1+|y|^{2}} & =\frac{1}{2} \int_{0}^{\infty}\left(1-e^{-\lambda|y|^{2} / 2}\right) e^{-\lambda / 2} d \lambda \\
& =\frac{1}{2} \int_{0}^{\infty} \int_{\mathbb{R}^{d}}(2 \pi \lambda)^{-n / 2}\left(1-e^{i y \cdot p}\right) e^{-|p|^{2} / 2 \lambda} e^{-\lambda / 2} d p d \lambda \\
& =\int_{0}^{\infty}\left(1-e^{i y \cdot p}\right) g(p) d p .
\end{aligned}
$$

The assertion follows since the left-hand side is real-valued. 
2.3. The Chernoff theorem. If $X, X_{1}, X_{2}$ are Banach spaces, then $L\left(X_{1}, X_{2}\right)$ denotes the space of continuous linear mappings from $X_{1}$ to $X_{2}$ equipped with the strong operator topology, $L(X)=L(X, X),\|\cdot\|$ denotes the operator norm on $L(X)$ and Id the identity operator in $X$. If $D(T) \subset X$ is a linear subspace and $T: D(T) \rightarrow X$ is a linear operator, then $D(T)$ denotes the domain of $T$.

The derivative at the origin of a function $F:[0, \varepsilon) \rightarrow L(X), \varepsilon>0$, is a linear mapping $F^{\prime}(0): D\left(F^{\prime}(0)\right) \rightarrow X$ such that

$$
F^{\prime}(0) g:=\lim _{t \searrow 0} \frac{F(t) g-F(0) g}{t},
$$

where $D\left(F^{\prime}(0)\right)$ is the vector space of all elements $g \in X$ for which the above limit exists.

In the sequel we use the following version of Chernoff's theorem (see [33]).

Theorem 2.4 (Chernoff). Let $X$ be a Banach space, $F:[0, \infty) \rightarrow L(X)$ be a (strongly) continuous mapping such that $F(0)=\operatorname{Id}$ and $\|F(t)\| \leq e^{a t}$ for some $a \in$ $[0, \infty)$ and all $t \geq 0$. Let $D$ be a linear subspace of $D\left(F^{\prime}(0)\right)$ such that the restriction of the operator $F^{\prime}(0)$ to this subspace is closable. Let $(A, D(A))$ be this closure. If $(A, D(A))$ is the generator of a strongly continuous semigroup $\left(T_{t}\right)_{t \geq 0}$, then for any $t_{0}>0$ the sequence $\left.(F(t / n))^{n}\right)_{n \in \mathbb{N}}$ converges to $\left(T_{t}\right)_{t>0}$ as $n \rightarrow \infty$ in the strong operator topology, uniformly with respect to $t \in\left[0, t_{0}\right]$, i.e., $T_{t}=\lim _{n \rightarrow \infty}(F(t / n))^{n}$.

A family of operators $(F(t))_{t \geq 0}$ is called Chernoff equivalent to the semigroup $\left(T_{t}\right)_{t \geq 0}$ if this family satisfies the assertions of Chernoff's theorem; then, by Chernoff's theorem we have in $L(X)$ locally uniformly with respect to $t$

$$
T_{t}=\lim _{n \rightarrow \infty}(F(t / n))^{n} .
$$

The equality (9) is called Feynman formula for the semigroup $\left(T_{t}\right)_{t \geq 0}$.

\section{Hamiltonian Feynman formula for some Feller semigroups}

Consider a function $H: \mathbb{R}^{d} \times \mathbb{R}^{d} \rightarrow \mathbb{C}$ which is measurable, locally bounded in both variables $(q, p)$, and satisfies for fixed $q$ the Lévy-Khintchine representation (6), i.e. $H(q, \cdot)$ is a continuous negative definite function for all $q \in \mathbb{R}^{d}$. Assume that

$$
\begin{gathered}
\sup _{q \in \mathbb{R}^{d}}|H(q, p)| \leqslant \kappa\left(1+|p|^{2}\right) \quad \text { for all } p \in \mathbb{R}^{d} \text { and some } \kappa>0, \\
\left.p \mapsto H(q, p) \quad \text { is uniformly (w.r.t. } q \in \mathbb{R}^{d}\right) \text { continuous at } p=0, \\
q \mapsto H(q, p) \quad \text { is continuous for all } p \in \mathbb{R}^{d} .
\end{gathered}
$$
have

$$
H(q, D) \varphi(q)=(2 \pi)^{-d / 2} \int_{\mathbb{R}^{d}} e^{i p \cdot q} H(q, p) \widehat{\varphi}(p) d p .
$$

Note that (due to the estimate (2)) the condition (10) actually means that a $\Psi$ DO $H(\cdot, D)$ is an operator with bounded "coefficients" $(a(q), \ell(q), Q(q), N(q, \cdot))$.

\section{Assumption A.}

(i) We assume that the function $H(q, p)$ is such that $-H(\cdot, D)$ is closable and the closure is the generator of a strongly continuous semigroup on $C_{\infty}\left(\mathbb{R}^{d}\right)$.

(ii) We assume also that the set $C_{c}^{\infty}\left(\mathbb{R}^{d}\right)$ of test functions is an operator core for this generator. 
Remark 3.1. Conditions on the function $H(q, p)$ to fulfill Assumption A (i) can be found, for example, in Vol. 2 of [22] (Thms. 2.6.4, 2.6.9, 2.7.9, 2.7.16, 2.7.19, 2.8.1) or in [24]. For all these constructions $C_{c}^{\infty}\left(\mathbb{R}^{d}\right)$ is always an operator core. Note that Assumption A (ii) holds also for example for generators of Lévy processes, see [29] (Theo. 31.5).

Let $F(t)$ be a $\Psi D O$ with the symbol $e^{-t H(q, p)}$, i.e. for each $\varphi \in C_{c}^{\infty}\left(\mathbb{R}^{d}\right)$

$$
F(t) \varphi(q)=(2 \pi)^{-d / 2} \int_{\mathbb{R}^{d}} e^{i p \cdot q} e^{-t H(q, p)} \widehat{\varphi}(p) d p .
$$

Lemma 3.1. For each $\varphi \in C_{c}^{\infty}\left(\mathbb{R}^{d}\right)$ the function $F(t) \varphi$ belongs to $C_{\infty}\left(\mathbb{R}^{d}\right)$.

Proof. The Fourier transform $\widehat{\varphi}$ of a test function $\varphi \in C_{c}^{\infty}\left(\mathbb{R}^{d}\right)$ is in the Schwartz space $S\left(\mathbb{R}^{d}\right)$ of rapidly decreasing functions. Since $q \mapsto e^{-t H(q, p)}$ is continuous (by assumption (12) ) and bounded ( $\operatorname{Re} H \geq 0$ due to properties of continuous negative definite functions), Lebesgue's Dominated Convergence theorem shows that $F(t)$ maps $C_{c}^{\infty}\left(\mathbb{R}^{d}\right)$ into $C\left(\mathbb{R}^{d}\right)$.

Let us prove, that $F(t) \varphi(q) \rightarrow 0$ when $|q| \rightarrow \infty$. Since $H(q, \cdot)$ is continuous negative definite for all $q \in \mathbb{R}^{d}$ then $e^{-t H(q, \cdot)}$ is continuous positive definite for all $q \in \mathbb{R}^{d}$ and all $t>0$ due to Theorem 2.1. Then the function

$$
\left[p \mapsto h_{t}(q, p):=e^{-t H(q, 0)}-e^{-t H(q, p)}\right]
$$

is also continuous negative definite for all $q \in \mathbb{R}^{d}$ by Corollary 2.1. Hence, $h_{t}(q, \cdot)$ satisfies a Lévy-Khintchine representation

(15) $h_{t}(q, p)=a_{t}(q)+i \ell_{t}(q) \cdot p+p \cdot Q_{t}(q) p+\int_{y \neq 0}\left(1-e^{i y \cdot p}+\frac{i y \cdot p}{1+|y|^{2}}\right) N_{t}(q, d y)$,

where $\left(a_{t}(q), \ell_{t}(q), Q_{t}(q), N_{t}(q, \cdot)\right)$ is for each $q \in \mathbb{R}^{d}$ the Lévy characteristics of $h_{t}(q, \cdot)$. Again we can consider a $\Psi$ DO $h_{t}(q, D)$ with the symbol $h_{t}(q, p)$, i.e. for each $\varphi \in C_{c}^{\infty}\left(\mathbb{R}^{d}\right)$

$$
\begin{aligned}
h_{t}(q, D) \varphi(q)= & (2 \pi)^{-d / 2} \int_{\mathbb{R}^{d}} e^{i p \cdot q} h_{t}(q, p) \widehat{\varphi}(p) d p \\
= & -a_{t}(q) \varphi(q)+\ell_{t}(q) \cdot \nabla \varphi(q)+\sum_{j, k=1}^{d} Q_{t}^{j k}(q) \partial_{j} \partial_{k} u(q) \\
& \quad+\int_{y \neq 0}\left(\varphi(q+y)-\varphi(q)-\frac{y \cdot \nabla \varphi(q)}{1+|y|^{2}}\right) N_{t}(q, d y)
\end{aligned}
$$

Note, that

$$
F(t) \varphi(q)=(2 \pi)^{-d / 2} e^{-t H(q, 0)} \int_{\mathbb{R}^{d}} e^{i p \cdot q} \widehat{\varphi}(p) d p-(2 \pi)^{-d / 2} \int_{\mathbb{R}^{d}} e^{i p \cdot q} h_{t}(q, p) \widehat{\varphi}(p) d p .
$$

Since $\operatorname{Re} H \geq 0$ then $\sup _{q \in \mathbb{R}^{d}}\left|e^{-t H(q, 0)}\right| \leq 1$, and the first integral in the above formula tends to zero as $|q| \rightarrow \infty$ by the Riemann-Lebesgue Theorem. Thus, we only need to show that

$$
\left[q \mapsto(2 \pi)^{-d / 2} \int_{\mathbb{R}^{d}} e^{i p \cdot q} h_{t}(q, p) \widehat{\varphi}(p) d p\right] \in C_{\infty}\left(\mathbb{R}^{d}\right)
$$


As $\varphi$ has compact support, there is some $R>0$ such that $\operatorname{supp} \varphi \subset B_{R}(0)$. For all $|q|>2 R$ formula (16) becomes

$$
\begin{aligned}
\left|h_{t}(q, D) \varphi(q)\right| & =\left|\int_{y \neq 0} \varphi(q+y) N_{t}(q, d y)\right| \\
& =\left|\int_{|y|>R} \varphi(q+y) N_{t}(q, d y)\right| \\
& \leqslant 2 \int_{y \neq 0} \frac{|y / R|^{2}}{1+|y / R|^{2}} N_{t}(q, d y) \cdot\|\varphi\|_{\infty} .
\end{aligned}
$$

The last line follows from the elementary inequality $\frac{1}{2} \leqslant \frac{t^{2}}{1+t^{2}}$ for $|t|>1$ which applies if $|y|>R$, and from $\varphi(q+y)=0$ if $|q|>2 R$ and $|y| \leqslant R$. We can now use Lemma 2.2, the Lévy-Khintchine representation of $h_{t}(q, \cdot)$ and the estimate (2) for a continuous negative definite function $h_{t}(q, \dot{\bar{R}})$ to get

$$
\begin{aligned}
\left|h_{t}(q, D) \varphi(q)\right| & \leqslant 2 \int_{y \neq 0} \int_{\mathbb{R}^{d}}\left(1-\cos \frac{y \cdot \eta}{R}\right) g(\eta) d \eta N_{t}(q, d y) \cdot\|\varphi\|_{\infty} \\
& \leq 2 \int_{\mathbb{R}^{d}} \operatorname{Re} h_{t}\left(q, \frac{\eta}{R}\right) g(\eta) d \eta \cdot\|\varphi\|_{\infty} \\
& \leqslant 2 \int_{\mathbb{R}^{d}}\left|h_{t}\left(q, \frac{\eta}{R}\right)\right| g(\eta) d \eta \cdot\|\varphi\|_{\infty} \\
& \leqslant 2 \sup _{|\xi| \leqslant 1 / R}\left|h_{t}(q, \xi)\right| \int_{\mathbb{R}^{d}}\left(1+|\eta|^{2}\right) g(\eta) d \eta \cdot\|\varphi\|_{\infty} .
\end{aligned}
$$

Since $g(\eta)$ has absolute moments of any order, we see

$$
\left|h_{t}(q, D) \varphi(q)\right| \leqslant c_{g} \sup _{q \in \mathbb{R}^{d}} \sup _{|\xi| \leqslant 1 / R}\left|h_{t}(q, \xi)\right| \cdot\|\varphi\|_{\infty} \quad \text { for all }|q|>2 R .
$$

As $h_{t}(q, 0)=0$, the condition (11) tells us that $\lim _{|q| \rightarrow \infty} h_{t}(q, D) \varphi(q)=0$. Therefore, $h_{t}(q, \cdot) \varphi \in C_{\infty}\left(\mathbb{R}^{d}\right)$.

Lemma 3.2. For each $t>0$ a mapping $F(t)$ can be extended to a contraction $F(t): C_{\infty}\left(\mathbb{R}^{d}\right) \rightarrow C_{\infty}\left(\mathbb{R}^{d}\right)$.

Proof. Let us freeze the coefficients (see e.g. [23]). For each $t>0$ and each $q_{0} \in \mathbb{R}^{d}$ let us consider a $\Psi \mathrm{DO} F^{q_{0}}(t)$ with the symbol $e^{-t H\left(q_{0}, p\right)}$, i.e. for any $\varphi \in C_{c}^{\infty}\left(\mathbb{R}^{d}\right)$ we have

$$
F^{q_{0}}(t) \varphi(q)=(2 \pi)^{-d / 2} \int_{\mathbb{R}^{d}} e^{i p \cdot q} e^{-t H\left(q_{0}, p\right)} \widehat{\varphi}(p) d p .
$$

Then $F(t) \varphi(q)=F^{q}(t) \varphi(q)$ for any $\varphi \in C_{c}^{\infty}\left(\mathbb{R}^{d}\right)$ and any $q \in \mathbb{R}^{d}$. Since for each $q_{0} \in \mathbb{R}^{d}$ the function $e^{-t H\left(q_{0}, \cdot\right)}$ is positive definite then there exists a convolution semigroup $\left(\mu_{t}^{q_{0}}\right)_{t \geq 0}$, such that $\mathcal{F}^{-1}\left[\mu_{t}^{q_{0}}\right]=(2 \pi)^{-d / 2} e^{-t H\left(q_{0}, \cdot\right)}$ and $F^{q_{0}}(t) \varphi(q)=$ $\int_{\mathbb{R}^{d}} \varphi(q-y) \mu_{t}^{q_{0}}(d y)$. Hence, for each $q_{0} \in \mathbb{R}^{d}$ a family $\left(F^{q_{0}}(t)\right)_{t \geq 0}$ is a Feller semigroup, and for each $q, q_{0} \in \mathbb{R}^{d}$ we have

$$
\left|F^{q_{0}}(t) \varphi(q)\right|=\left|\int_{\mathbb{R}^{d}} \varphi(q-y) \mu_{t}^{q_{0}}(d y)\right| \leq\|\varphi\|_{\infty} .
$$


Then $\|F(t) \varphi\|_{\infty}=\sup _{q \in \mathbb{R}^{d}}|F(t) \varphi(q)|=\sup _{q \in \mathbb{R}^{d}}\left|F^{q}(t) \varphi(q)\right| \leq\|\varphi\|_{\infty}$ for any $\varphi \in$ $S\left(\mathbb{R}^{d}\right)$. Hence, the family $F(t)$ can be extended to a contraction from $C_{\infty}\left(\mathbb{R}^{d}\right)$ into itself by the B.L.T. Theorem (see [28, p.9).

Theorem 3.1. Let the function $H: \mathbb{R}^{d} \times \mathbb{R}^{d} \rightarrow \mathbb{C}$ be measurable and locally bounded in both variables $(q, p)$. Assume that $H(q, \cdot)$ is continuous and negative definite for all $q \in \mathbb{R}^{d}$ and that conditions (10), (11) and (12) hold. Under Assumption $A$ the family $(F(t))_{t \geq 0}$ is Chernoff equivalent to a strongly continuous semigroup $\left(T_{t}\right)_{t \geq 0}$, generated by the closure of the $\Psi D O-H(\cdot, D)$ with the symbol $-H(q, p)$, and the Hamiltonian Feynman formula $T_{t}=\lim _{n \rightarrow \infty}\left[F\left(\frac{t}{n}\right)\right]^{n}$ is valid in $L\left(C_{\infty}\left(\mathbb{R}^{d}\right)\right)$ locally uniformly with respect to $t \geq 0$.

Proof. By Lemma 3.2 each $F(t)$ is a contraction operator on $C_{\infty}\left(\mathbb{R}^{d}\right)$, thus we only need to prove that for all $\varphi \in C_{\infty}\left(\mathbb{R}^{d}\right)$ we have $\lim _{t \rightarrow 0}\|F(t) \varphi-\varphi\|_{\infty}=0$ and $F^{\prime}(0)=-H(\cdot, D)$ on a core of $-H(\cdot, D)$.

For any $\varphi \in C_{c}^{\infty}\left(\mathbb{R}^{d}\right)$ we have, due to the estimate (10),

$$
\begin{aligned}
\lim _{t \rightarrow 0}\|F(t) \varphi-\varphi\|_{\infty} & =\lim _{t \rightarrow 0} \sup _{q \in \mathbb{R}^{d}}\left|(2 \pi)^{-d / 2} \int_{\mathbb{R}^{d}} e^{i p \cdot q} \widehat{\varphi}(p)\left[e^{-t H(q, p)}-1\right] d p\right| \\
& \leq \lim _{t \rightarrow 0}(2 \pi)^{-d / 2} \int_{\mathbb{R}^{d}}|\widehat{\varphi}(p)| \sup _{q \in \mathbb{R}^{d}}\left\{\left|\frac{e^{-t H(q, p)}-1}{-t H(q, p)}\right||t H(q, p)|\right\} d p \\
& \leq \lim _{t \rightarrow 0}(2 \pi)^{-d / 2} \int_{\mathbb{R}^{d}} t \kappa\left(1+|p|^{2}\right)|\widehat{\varphi}(p)| d p \\
& =0
\end{aligned}
$$

since $\widehat{\varphi} \in S\left(\mathbb{R}^{d}\right)$. Hence, $\lim _{t \rightarrow 0}\|F(t) \varphi-\varphi\|_{\infty}=0$ for all $\varphi \in C_{c}^{\infty}\left(\mathbb{R}^{d}\right)$. As $\|F(t)\| \leq$ 1 , then the last equality is true for all $\varphi \in C_{\infty}\left(\mathbb{R}^{d}\right)$ by a 3 -epsilon argument.

In a similar way, for any $\varphi \in C_{c}^{\infty}\left(\mathbb{R}^{d}\right)$ we have

$$
\begin{aligned}
\lim _{t \rightarrow 0} \| \frac{F(t) \varphi-\varphi}{t} & +H(\cdot, D) \varphi \|_{\infty} \\
& =\lim _{t \rightarrow 0} \sup _{q \in \mathbb{R}^{d}}\left|(2 \pi)^{-d / 2} \int_{\mathbb{R}^{d}} e^{i p \cdot q} \widehat{\varphi}(p)\left[\frac{e^{-t H(q, p)}-1}{t}+(H(q, p))\right] d p\right| \\
& \leq \lim _{t \rightarrow 0}(2 \pi)^{-d / 2} \int_{\mathbb{R}^{d}}|\widehat{\varphi}(p)| \frac{t \kappa^{2}\left(1+|p|^{2}\right)^{2}}{2} d p \\
& =0 .
\end{aligned}
$$

Thus, all assumptions of Chernoff's theorem are fulfilled, and the family $F(t)$ is Chernoff equivalent to the semigroup $T_{t}$ generated by $-H(\cdot, D)$.

Remark 3.2. (i) Let us assume additionally that $H: \mathbb{R}^{d} \times \mathbb{R}^{d} \rightarrow \mathbb{C}$ satisfies the following condition:

$$
\exists C>0 \text { such that }\left\|\partial_{q}^{\alpha} \partial_{p}^{\beta} e^{t H}\right\|_{L^{\infty}\left(\mathbb{R}^{d} \times \mathbb{R}^{d}\right)} \leq C,
$$

where $\alpha, \beta \in \mathbb{N}_{0}^{d}, \alpha=0$ or $1, \beta=0$ or $1, \partial_{q}^{\alpha} \partial_{p}^{\beta}$ are derivatives in the distributional sense. Note, that this condition is fulfilled, e.g. if $H:|H(q, p)| \geq c|p|^{r}$ for $|p| \gg 1$, some $c>0$ and some $r \in(0,2)$. Then by Theorem 2 of Ref. [19] we have $F(t): L_{2}\left(\mathbb{R}^{d}\right) \rightarrow L_{2}\left(\mathbb{R}^{d}\right)$. In this case the Hamiltonian Feynman formula obtained 
in Theorem 3.1 has the following form:

(17)

$$
\begin{aligned}
& \left(T_{t} \varphi\right)\left(q_{0}\right) \\
& =\lim _{n \rightarrow \infty} \frac{1}{(2 \pi)^{d n}} \int_{\left(\mathbb{R}^{d}\right)^{2 n}} e^{i \sum_{k=1}^{n} p_{k} \cdot\left(q_{k-1}-q_{k}\right)} e^{-\frac{t}{n} \sum_{k=1}^{n} H\left(q_{k-1}, p_{k}\right)} \varphi\left(q_{n}\right) d q_{1} d p_{1} \cdots d q_{n} d p_{n},
\end{aligned}
$$

where the equality holds in $L_{2}$-sense (i.e. the integrals in the right hand side must be considered in a regularized sense). We refer to [19] for further conditions on $H(q, p)$ ensuring $F(t): L_{2}\left(\mathbb{R}^{d}\right) \rightarrow L_{2}\left(\mathbb{R}^{d}\right)$.

(ii) If the function $H$ satisfies sufficient conditions for $F(t) \varphi$ to be in $S\left(\mathbb{R}^{d}\right)$ for each $\varphi \in S\left(\mathbb{R}^{d}\right)$ then for any $\varphi \in S\left(\mathbb{R}^{d}\right)$ the equality in the Hamiltonian Feynman formula (17) holds in each point $q_{0} \in \mathbb{R}^{d}$. Such conditions can be found in the following lemma.

Lemma 3.3. Let $H: \mathbb{R}^{d} \times \mathbb{R}^{d} \rightarrow \mathbb{C}$ be a continuous function such that for each $q \in \mathbb{R}^{d}$ a mapping $p \mapsto H(q, p)$ is negative definite and $H(\cdot, \cdot) \in C^{\infty}\left(\mathbb{R}^{d} \times \mathbb{R}^{d}\right)$. Assume that for each $p \in \mathbb{R}^{d}$ and for each $\alpha, \beta \in \mathbb{N}_{0}^{d}$, the following estimates hold:

$$
\sup _{q \in \mathbb{R}^{d}}\left|\partial_{p}^{\alpha} \partial_{q}^{\beta} H(q, p)\right| \leq f_{\alpha, \beta}(p),
$$

where all functions $f_{\alpha, \beta}$ are continuous on $\mathbb{R}^{d}$ and have at most polynomial growth at infinity. Then $F(t) \varphi \in S\left(\mathbb{R}^{d}\right)$ for each $\varphi \in S\left(\mathbb{R}^{d}\right)$.

Proof. By Lemma 3.2 we have $F(t) \varphi \in C_{\infty}\left(\mathbb{R}^{d}\right)$. Let us show that for all $\alpha, \beta \in \mathbb{N}_{0}^{d}$ the norm

$$
\|F(t) \varphi\|_{\alpha, \beta}=\sup _{q \in \mathbb{R}^{d}}\left|q^{\alpha} \partial_{q}^{\beta}[F(t) \varphi](q)\right|
$$

is finite. Note that for any $\beta \in \mathbb{N}_{0}^{d}$ the function $\partial_{q}^{\beta} e^{-t H(q, p)+i p \cdot q}$ is continuous. By (18) it is also majorized (uniformly for all $q$ ) by some continuous function of $p$ which has at most polynomial growth at infinity. Hence, by Lebesgue's dominated convergence theorem, we have

$$
\begin{aligned}
q^{\alpha} \partial_{q}^{\beta}[F(t) \varphi](q) & =(2 \pi)^{-d / 2} \int_{\mathbb{R}^{d}} q^{\alpha} \partial_{q}^{\beta} e^{-t H(q, p)+i p \cdot q} \widehat{\varphi}(p) d p \\
& =(2 \pi)^{-d / 2} \sum_{0 \leq \gamma \leq \beta} \int_{\mathbb{R}^{d}} q^{\alpha} \partial_{q}^{\gamma}\left(e^{i p \cdot q}\right) \partial_{q}^{\beta-\gamma}\left(e^{-t H(q, p)}\right) \widehat{\varphi}(p) d p .
\end{aligned}
$$

Since $\partial_{q}^{\gamma}\left(e^{i p \cdot q}\right)=e^{i p \cdot q} R_{\gamma}(p)$, where $R_{\gamma}$ is a polynomial of $p$, we can use integration by parts and get

$$
\begin{aligned}
q^{\alpha} \partial_{q}^{\beta}[F(t) \varphi](q) & =(2 \pi)^{-d / 2} \sum_{0 \leq \gamma \leq \beta} \int_{\mathbb{R}^{d}} q^{\alpha} e^{i p \cdot q}\left[R_{\gamma}(p) \partial_{q}^{\beta-\gamma}\left(e^{-t H(q, p)}\right) \widehat{\varphi}(p)\right] d p \\
& =(2 \pi)^{-d / 2} \sum_{0 \leq \gamma \leq \beta} i^{|\alpha|} \int_{\mathbb{R}^{d}} \partial_{p}^{\alpha} e^{i p \cdot q}\left[R_{\gamma}(p) \partial_{q}^{\beta-\gamma}\left(e^{-t H(q, p)}\right) \widehat{\varphi}(p)\right] d p \\
& =(2 \pi)^{-d / 2} \sum_{0 \leq \gamma \leq \beta}(-i)^{|\alpha|} \int_{\mathbb{R}^{d}} e^{i p \cdot q} \partial_{p}^{\alpha}\left[R_{\gamma}(p) \partial_{q}^{\beta-\gamma}\left(e^{-t H(q, p)}\right) \widehat{\varphi}(p)\right] d p
\end{aligned}
$$

Since $\partial_{p}^{\alpha}\left[R_{\gamma}(p) \partial_{q}^{\beta-\gamma}\left(e^{-t H(q, p)}\right) \widehat{\varphi}(p)\right]$ is bounded by an $L_{1}$-function which is independent of $q$, we can use (18) to see that the expression in the last line is finite. Hence, the norm $\|F(t) \varphi\|_{\alpha, \beta}$ is finite. 
Remark 3.3. If in Assumption A (i) we require the existence of not just a strongly continuous but a Feller semigroup, we obtain a Hamiltonian Feynman formula for the corresponding Feller process $\xi_{t}$. Besides, for each fixed $n$ the operator $[F(t / n)]^{n}$ in the Hamiltonian Feynman formula corresponds to the approximation of the process $\xi_{t}$ by a Markov chain $\left\{Y^{t / n}(k)\right\}_{k=0}^{n}$ with Lévy increments. This Markov chain is obtained by splitting the time interval $[0, t]$ onto $n$ equal steps and "freezing" the coefficient $q$ in the transition probabilities of $\xi_{t}$ at each step (cf. 4]). Moreover, the transition kernels $\mu_{q, t / n}$ of this Markov chain correspond to the transition operator $W_{t / n}, W_{t / n} \varphi(q)=\int_{\mathbb{R}^{d}} \varphi(y) \mu_{q, t / n}(d y)$. Hence, $[F(t / n)]^{n}=\left[W_{t / n}\right]^{n}$. This allows us to transform the obtained Hamiltonian Feynman formula for the Feller semigroup $T_{t}$ associated with the process $\xi_{t}$ into a Lagrangian Feynman formula $T_{t} \varphi(q)=\lim _{n \rightarrow \infty}\left[W_{t / n}\right]^{n} \varphi(q)$.

Example 3.1. Let us consider the symbol $H_{1}(q, p)=a(q)|p|^{\alpha}$, where $\alpha \in(0,2]$ and $a(\cdot) \in C^{\infty}\left(\mathbb{R}^{d}\right)$ is a strictly positive and bounded function. Then $-H_{1}(\cdot, D)$ generates a Feller semigroup $\left(T_{t}^{1}\right)_{t \geq 0}$ (see [31]). If $\alpha=2$ this semigroup corresponds to the process of diffusion with variable diffusion coefficient. All conditions of the Theorem 3.1 are fulfilled and by the Hamiltonian Feynman formula (17) for any $\varphi \in C_{c}^{\infty}\left(\mathbb{R}^{d}\right)$ and any $q_{0} \in \mathbb{R}^{d}$ we have:

$$
\begin{aligned}
& \left(T_{t}^{1} \varphi\right)\left(q_{0}\right) \\
& =\lim _{n \rightarrow \infty} \frac{1}{(2 \pi)^{d n}} \int_{\mathbb{R}^{2 d n}} e^{i \sum_{k=1}^{n} p_{k} \cdot\left(q_{k-1}-q_{k}\right)} e^{-\frac{t}{n} \sum_{k=1}^{n} a\left(q_{k-1}\right)\left|p_{k}\right|^{\alpha}} \varphi\left(q_{n}\right) d q_{1} d p_{1} \cdots d q_{n} d p_{n} .
\end{aligned}
$$

Example 3.2. Let us consider the symbol $H_{2}(q, p)=\sqrt{|p|^{\alpha}+m^{2}(q)}-m(q)$, where $m(\cdot) \in C^{\infty}\left(\mathbb{R}^{d}\right)$ is a strictly positive and bounded function on $\mathbb{R}^{d}, \alpha \in(0,2]$. If additionally the function $m(\cdot)$ is such that the Assumption A holds (e.g. if $m \equiv$ const), then the following Hamiltonian Feynman formula is valid for the corresponding semigroup $\left(T_{t}^{2}\right)_{t \geq 0}$ :

$$
\begin{array}{r}
\left(T_{t}^{2} \varphi\right)\left(q_{0}\right)=\lim _{n \rightarrow \infty} \frac{1}{(2 \pi)^{d n}} \int_{\mathbb{R}^{2 d n}} e^{i \sum_{k=1}^{n} p_{k} \cdot\left(q_{k-1}-q_{k}\right)} e^{-\frac{t}{n} \sum_{k=1}^{n} \sqrt{\left|p_{k}\right|^{\alpha}+m^{2}\left(q_{k-1}\right)}-m\left(q_{k-1}\right)} \times \\
\times \varphi\left(q_{n}\right) d q_{1} d p_{1} \cdots d q_{n} d p_{n}
\end{array}
$$

where $\varphi \in C_{c}^{\infty}\left(\mathbb{R}^{d}\right)$ and $q_{0} \in \mathbb{R}^{d}$. In the case $\alpha=2$ the operator $H_{2}(\cdot, D)$ can be considered as a Hamiltonian of a free relativistic (quasi-)particle with variable mass (cf. [18, 20]).

\section{Lagrangian Feynman formula for multiplicative perturbations of Feller semigroups}

Let $H(\cdot, D)$ be a $\Psi$ DO with the symbol $H(q, p)$. Assume that $-H(\cdot, D)$ generates a Feller semigroup $\left(T_{t}\right)_{t \geq 0}$,

$$
T_{t} \varphi(q)=\int_{\mathbb{R}^{d}} \varphi(y) p_{t}(q, d y),
$$

where $p_{t}(q, d y)=\mathbb{P}^{q}\left(X_{t} \in d y\right)$ is the transition probability of the underlying Feller process $X_{t}, \varphi \in C_{\infty}\left(\mathbb{R}^{d}\right)$. Let $a(\cdot): \mathbb{R}^{d} \rightarrow[c, 1 / c], c>0$, be a continuous function. Then (due to [14]) a $\Psi$ DO $-\widetilde{H}(\cdot, D)$ with the symbol $-\widetilde{H}(q, p)=-a(q) H(q, p)$ generates a strongly continuous semigroup $\left(\widetilde{T}_{t}\right)_{t \geq 0}$ on the space $C_{\infty}\left(\mathbb{R}^{d}\right)$. 
Consider now a family $(\widetilde{F}(t))_{t \geq 0}$ of operators on $C_{\infty}\left(\mathbb{R}^{d}\right)$ defined by the formula:

$$
\widetilde{F}(t) \varphi(q)=\left(T_{a(q) t} \varphi\right)(q) \equiv \int_{\mathbb{R}^{d}} \varphi(y) p_{a(q) t}(q, d y)
$$

Theorem 4.1. The family $(\widetilde{F}(t))_{t \geq 0}$ given by the formula (19) is Chernoff equivalent to the semigroup $\left(\widetilde{T}_{t}\right)_{t \geq 0}$ generated by a $\Psi D O-\widetilde{H}(\cdot, D)$ with the symbol $-\widetilde{H}(q, p)=-a(q) H(q, p)$ and, hence, the Lagrangian Feynman formula

$$
\begin{array}{r}
\widetilde{T}_{t} \varphi\left(q_{0}\right)=\lim _{n \rightarrow \infty} \int_{\mathbb{R}^{d}} \cdots \int_{\mathbb{R}^{d}} \varphi\left(q_{n}\right) p_{a\left(q_{0}\right) t / n}\left(q_{0}, d q_{1}\right) p_{a\left(q_{1}\right) t / n}\left(q_{1}, d q_{2}\right) \cdots \\
\cdots p_{a\left(q_{n-1}\right) t / n}\left(q_{n-1}, d q_{n}\right)
\end{array}
$$

is valid in $L\left(C_{\infty}\left(\mathbb{R}^{d}\right)\right)$ locally uniformly with respect to $t \geq 0$.

Remark 4.1. The transformation of a Feller process under which a symbol $H(q, p)$ transfers into a symbol $a(q) H(q, p)$ may be understood as a position-dependent time re-scaling of the process: $t \rightsquigarrow a(q) t$. Indeed, for each random variable $X_{a(q) t}$ we have:

$$
\frac{\mathbb{E}^{q}\left[e^{i\left(X_{a(q) t}-q\right) \cdot p}\right]-1}{t}=a(q) \frac{\mathbb{E}^{q}\left[e^{i\left(X_{a(q) t}-q\right) \cdot p}\right]-1}{a(q) t} \longrightarrow-a(q) H(q, p), \quad t \rightarrow 0 .
$$

Note, that $p(t, x, d y)=p_{a(x) t}(x, d y)$ is NOT the transition function of the re-scaled process.

If the explicit form of the transition density of the original Feller process is known then the Lagrangian Feynman formula (20) for the time re-scaled process contains only explicit - usually elementary - functions.

Proof. First, let us prove that the family $(\widetilde{F}(t))_{t \geq 0}$ acts in the space $C_{\infty}\left(\mathbb{R}^{d}\right)$. For any fixed $\varphi \in C_{\infty}\left(\mathbb{R}^{d}\right)$ we have

$$
\begin{aligned}
\lim _{q \rightarrow q_{0}} \mid & \tilde{F}(t) \varphi(q)-\tilde{F}(t) \varphi\left(q_{0}\right) \mid \\
& =\lim _{q \rightarrow q_{0}}\left|\left(T_{a(q) t} \varphi\right)(q)-\left(T_{a\left(q_{0}\right) t} \varphi\right)\left(q_{0}\right)\right| \\
& \leq \lim _{q \rightarrow q_{0}}\left(\left|\left(T_{a(q) t} \varphi\right)(q)-\left(T_{a\left(q_{0}\right) t} \varphi\right)(q)\right|+\left|\left(T_{a\left(q_{0}\right) t} \varphi\right)(q)-\left(T_{a\left(q_{0}\right) t} \varphi\right)\left(q_{0}\right)\right|\right) \\
& \leq \lim _{q \rightarrow q_{0}}\left\|\left[T_{a(q) t}-T_{a\left(q_{0}\right) t}\right] \varphi\right\|_{\infty}+\lim _{q \rightarrow q_{0}}\left|\left(T_{a\left(q_{0}\right) t} \varphi\right)(q)-\left(T_{a\left(q_{0}\right) t} \varphi\right)\left(q_{0}\right)\right| \\
& =0 .
\end{aligned}
$$

Therefore, the function $\widetilde{F}(t) \varphi$ is continuous. Since

$$
\begin{aligned}
\lim _{|q| \rightarrow \infty}|\widetilde{F}(t) \varphi(q)| & =\lim _{|q| \rightarrow \infty}\left|\left(T_{a(q) t} \varphi\right)(q)\right| \\
& \leq \lim _{|q| \rightarrow \infty} \sup _{q_{0} \in \mathbb{R}^{d}}\left|T_{a\left(q_{0}\right) t} \varphi(q)\right| \\
& =\lim _{|q| \rightarrow \infty} \sup _{s \in[c t, t / c]}\left|T_{s} \varphi(q)\right|,
\end{aligned}
$$

we get that $\tilde{F}(t): C_{\infty}\left(\mathbb{R}^{d}\right) \rightarrow C_{\infty}\left(\mathbb{R}^{d}\right)$.

Since the semigroup $\left(T_{t}\right)_{t \geq 0}$ acts in $C_{\infty}\left(\mathbb{R}^{d}\right)$, then for any $t \geq 0$ and $\varepsilon>0$ there exists $R_{\varepsilon, t}>0$ such that for any $q \in \mathbb{R}^{d}:|q|>R_{\varepsilon, t}$ the inequality $\left|T_{t} \varphi(q)\right|<\frac{1}{2} \varepsilon$ holds. Due to the strong continuity of $\left(T_{t}\right)_{t>0}$ there exists $\delta_{\varepsilon}>0$ such that for all $\tau, \tau^{\prime} \in[c t, t / c]$ with $\left|\tau-\tau^{\prime}\right|<\delta_{\varepsilon}$ the inequality $\left\|T_{\tau} \varphi-T_{\tau^{\prime}} \varphi\right\|_{\infty}<\frac{1}{2} \varepsilon$ holds. Let us fix $\varepsilon>0$. Consider a partition $\tau_{0}=c t<\tau_{1}<\ldots<\tau_{N}=t / c$ of a segment $[c t, t / c]$ such that $\max _{1 \leq k \leq N}\left|\tau_{k}-\tau_{k-1}\right|<\delta_{\varepsilon}$. Then for any $\tau \in[c t, t / c]$ ther exists $\tau_{k}$ 
with $\left|\tau-\tau_{k}\right|<\delta_{\varepsilon}$. Let now $R_{\varepsilon}=\max _{0 \leq k \leq N} R_{\varepsilon, \tau_{k}}$. Then for any $q \in \mathbb{R}^{d}:|q|>R_{\varepsilon}$ and any $\tau \in[c t, t / c]$ we have

$$
\begin{aligned}
\left|T_{\tau} \varphi(q)\right| & \leq\left|T_{\tau} \varphi(q)-T_{\tau_{k}} \varphi(q)\right|+\left|T_{\tau_{k}} \varphi(q)\right| \\
& \leq\left\|T_{\tau} \varphi-T_{\tau_{k}} \varphi\right\|_{\infty}+\left|T_{\tau_{k}} \varphi(q)\right| \\
& \leq \frac{1}{2} \varepsilon+\frac{1}{2} \varepsilon=\varepsilon .
\end{aligned}
$$

Therefore, $\lim _{|q| \rightarrow \infty} \sup _{\tau \in\left[c_{1} t, c_{2} t\right]}\left|T_{\tau} \varphi(q)\right|=0$ and, hence, the function $\widetilde{F}(t) \varphi$ vanishes at infinity.

Further we use a freezing-in technique. For each $q_{0} \in \mathbb{R}^{d}$ consider a family of operators $\left(F^{q_{0}}(t)\right)_{t \geq 0}$ on $C_{\infty}\left(\mathbb{R}^{d}\right)$ such that

$$
F^{q_{0}}(t) \varphi(q)=\int_{\mathbb{R}^{d}} \varphi(y) p_{a\left(q_{0}\right) t}(q, d y) \equiv\left(T_{a\left(q_{0}\right) t} \varphi\right)(q) .
$$

Then $\widetilde{F}(t) \varphi(q)=F^{q}(t) \varphi(q)$ for all $\varphi \in C_{\infty}\left(\mathbb{R}^{d}\right), q \in \mathbb{R}^{d}$. Moreover, $\widetilde{F}(0)=T_{0}=\mathrm{Id}$ and, as $\left(T_{t}\right)_{t \geq 0}$ is a contraction semigroup, we have

$$
\begin{aligned}
\|\widetilde{F}(t) \varphi\|_{\infty} & \leq \sup _{q_{0} \in \mathbb{R}^{d}} \sup _{q \in \mathbb{R}^{d}}\left|F^{q_{0}}(t) \varphi(q)\right| \\
& =\sup _{q_{0} \in \mathbb{R}^{d}}\left\|T_{a\left(q_{0}\right) t} \varphi\right\|_{\infty} \\
& \leq\|\varphi\|_{\infty} .
\end{aligned}
$$

The family $(\widetilde{F}(t))_{t \geq 0}$ is strongly continuous since

$$
\begin{aligned}
\lim _{t \rightarrow 0}\|\widetilde{F}(t) \varphi-\varphi\|_{\infty} & =\lim _{t \rightarrow 0} \sup _{q \in \mathbb{R}^{d}}\left|F^{q}(t) \varphi(q)-\varphi(q)\right| \\
& \leq \lim _{t \rightarrow 0} \sup _{q_{0} \in \mathbb{R}^{d}} \sup _{q \in \mathbb{R}^{d}}\left|F^{q_{0}}(t) \varphi(q)-\varphi(q)\right| \\
& =\lim _{t \rightarrow 0} \sup _{q_{0} \in \mathbb{R}^{d}}\left\|T_{a\left(q_{0}\right) t} \varphi-\varphi\right\|_{\infty} \\
& \leqslant \lim _{t \rightarrow 0} \sup _{a \in[c, 1 / c]}\left\|T_{a t} \varphi-\varphi\right\|_{\infty} \\
& =0 .
\end{aligned}
$$

And for all $\varphi \in D(-H(\cdot, D))$ we have

$$
\begin{aligned}
\| \frac{\widetilde{F}(t) \varphi-\varphi}{t} & +\widetilde{H}(\cdot, D) \varphi \|_{\infty} \\
& =\sup _{q \in \mathbb{R}^{d}}\left|\frac{F^{q}(t) \varphi(q)-\varphi(q)}{t}+a(q) H(q, D) \varphi(q)\right| \\
& \leq \sup _{q_{0} \in \mathbb{R}^{d}} \sup _{q \in \mathbb{R}^{d}}\left|\frac{F^{q_{0}}(t) \varphi(q)-\varphi(q)}{t}+a\left(q_{0}\right) H(q, D) \varphi(q)\right| \\
& =\sup _{q_{0} \in \mathbb{R}^{d}} \sup _{q \in \mathbb{R}^{d}}\left|\frac{-1}{a\left(q_{0}\right) t} \int_{0}^{a\left(q_{0}\right) t} a\left(q_{0}\right) H(q, D)\left(T_{\tau} \varphi(q)-\varphi(q)\right) d \tau\right| \\
& \leq \frac{1}{t} \int_{0}^{t / c}\left\|H(q, D)\left(T_{\tau} \varphi(q)-\varphi(q)\right)\right\|_{\infty} d \tau \\
& \longrightarrow 0, \quad t \rightarrow 0 .
\end{aligned}
$$


Therefore, all assumptions of Chernoff's theorem are fulfilled and, hence, the family $(\widetilde{F}(t))_{t \geq 0}$ is Chernoff equivalent to the semigroup $\left(\widetilde{T}_{t}\right)_{t \geq 0}$ generated by a $\Psi \mathrm{DO}$ $-\widetilde{H}(\cdot, D)=-a(\cdot) H(\cdot, D)$.

Remark 4.2. One can show that (the appropriate modification of) Theorem 4.1 remains true for multiplicative perturbations of not necessary Feller but just strongly continuous semigroups on $C_{\infty}\left(\mathbb{R}^{d}\right)$.

Remark 4.3. If the symbol $-H(q, p)$ of a $\Psi \mathrm{DO}-H(\cdot, D)$ generating a Feller semigroup $\left(T_{t}\right)_{t \geq 0}$ satisfies the assumptions of Theorem 3.1, then the symbol $-\widetilde{H}(q, p)=$ $-a(q) H(q, p)$ (with continuous $a(\cdot): \mathbb{R}^{d} \rightarrow[c, 1 / c], c>0$ ) also satisfies the assumptions of this Theorem. Hence, the Hamiltonian Feynman formula obtained in Theorem 3.1 remains valid for the perturbed semigroup $\left(\widetilde{T}_{t}\right)_{t \geq 0}$ as well.

Example 4.1 (diffusion with variable diffusion coefficient). Let $\psi(p)=\frac{1}{2}|p|^{2}$ be the characteristic exponent of a Brownian motion in $\mathbb{R}^{d}$. The generator of Brownian motion is $-\psi(D)=\frac{1}{2} \Delta$. The transition density is given by Gaussian density

$$
p_{t}^{B M}(x)=(2 \pi t)^{-d / 2} \exp \left\{-\frac{|x|^{2}}{2 t}\right\} .
$$

Consider the semigroup $\left(\widetilde{T}_{t}\right)_{t \geq 0}$, generated by a $\Psi$ DO $-\widetilde{H}(\cdot, D)$ with the symbol $-\widetilde{H}(q, p)=-\frac{1}{2} a(q)|p|^{2}$, where $a(\cdot)$ is as before. Then by Theorem 4.1 for each $\varphi \in C_{\infty}\left(\mathbb{R}^{d}\right)$ we have (cf. [7], [8]):

$$
\begin{aligned}
\widetilde{T}_{t} \varphi\left(q_{0}\right)=\lim _{n \rightarrow \infty} \int_{\mathbb{R}^{d}} & \cdots \int_{\mathbb{R}^{d}}\left(2 \pi a\left(q_{0}\right) t / n\right)^{-d / 2} \exp \left\{-\frac{\left|q_{0}-q_{1}\right|^{2}}{2 a\left(q_{0}\right) t / n}\right\} \cdots \\
& \cdots\left(2 \pi a\left(q_{n-1}\right) t / n\right)^{-d / 2} \exp \left\{-\frac{\left|q_{n-1}-q_{n}\right|^{2}}{2 a\left(q_{n-1}\right) t / n}\right\} \varphi\left(q_{n}\right) d q_{1} \cdots d q_{n}
\end{aligned}
$$

Example 4.2 (Cauchy type process with variable coefficient). Let $\psi(p)=|p|$ be the characteristic exponent of the Cauchy process in $\mathbb{R}^{d}$. The generator of the Cauchy process is $-\psi(D)=-\sqrt{-\Delta}$. The transition density is given by the formula

$$
p_{t}(x)=\Gamma\left(\frac{d}{2}+\frac{1}{2}\right) \frac{t}{\left[\pi|x|^{2}+t^{2}\right]^{(d+1) / 2}},
$$

where $\Gamma(\cdot)$ is Euler's Gamma function.

Consider the semigroup $\left(\widetilde{T}_{t}\right)_{t \geq 0}$, generated by a $\Psi \mathrm{DO}-\widetilde{H}(\cdot, D)$ with the symbol $-\widetilde{H}(q, p)=-a(q)|p|$, where $a(\cdot)$ is as before. Then by Theorem 4.1 for each $\varphi \in$ $C_{\infty}\left(\mathbb{R}^{d}\right)$ we have (cf. 9$]$ ):

$$
\begin{aligned}
\widetilde{T}_{t} \varphi\left(q_{0}\right)=\lim _{n \rightarrow \infty} \int_{\mathbb{R}^{d}} & \cdots \int_{\mathbb{R}^{d}}\left[\Gamma\left(\frac{d}{2}+\frac{1}{2}\right)\right]^{n} \frac{a\left(q_{0}\right) t / n}{\left[\left(a\left(q_{0}\right) t / n\right)^{2}+\left(\pi\left|q_{0}-q_{1}\right|\right)^{2}\right]^{(d+1) / 2}} \cdots \\
& \cdots \frac{a\left(q_{n-1}\right) t / n}{\left[\left(a\left(q_{n-1}\right) t / n\right)^{2}+\left(\pi\left|q_{n-1}-q_{n}\right|\right)^{2}\right]^{(d+1) / 2}} \varphi\left(q_{n}\right) d q_{1} \cdots d q_{n} .
\end{aligned}
$$

5. FEYNMAN FORMUlaE FOR ADDITIVE PERTURBATIONS OF SEMIGROUPS

Theorem 5.1. Let $X$ be a Banach space with a norm $\|\cdot\|_{X}$. Let $\left(T_{k}(t)\right)_{t>0}$, $k=1, \ldots, m$, be strongly continuous semigroups on $X$ with generators $\left(A_{k}, D\left(A_{k}\right)\right)$ respectively. Assume that $A=A_{1}+\cdots+A_{m}$ with domain $D(A)=\cap_{k=1}^{m} D\left(A_{k}\right)$ is closable and that the closure is the generator of a strongly continuous semigroup $(T(t))_{t \geq 0}$ on $X$. Let $\left(F_{k}(t)\right)_{t \geq 0}, k=1, \ldots, m$, be families of operators in $X$ which are Chernoff equivalent to the semigroups $\left(T_{k}(t)\right)_{t \geq 0}$ respectively, i.e. for each $k \in\{1, \ldots, m\}$ we have $F_{k}(0)=\mathrm{Id},\left\|F_{k}(t)\right\| \leq e^{a_{k} t}$ for some $a_{k}>0$ and there is $a$ 
set $D_{k} \subset D\left(A_{k}\right)$, which is a core for $A_{k}$, such that $\lim _{t \rightarrow 0}\left\|\frac{F_{k}(t) \varphi-\varphi}{t}-A_{k} \varphi\right\|_{X}=0$ for each $\varphi \in D_{k}$. Assume that there exists a set $D \subset \cap_{k=1}^{m} D_{k}$ which is a core for $A$. Then the family $(F(t))_{t \geq 0}$, where $F(t)=F_{1}(t) \circ \cdots \circ F_{m}(t)$ is Chernoff equivalent to the semigroup $(T(t))_{t \geq 0}$ and, hence, the Feynman formula

$$
T_{t}=\lim _{n \rightarrow \infty}[F(t / n)]^{n}
$$

is valid in the strong operator topology locally uniformly with respect to $t \geq 0$.

Proof. Obviously, the family $(F(t))_{t \geq 0}$ is strongly continuous, $F(0)=\mathrm{Id}$ and

$$
\|F(t)\| \leq\left\|F_{1}(t)\right\| \cdot \ldots \cdot\left\|F_{m}(t)\right\| \leq e^{\left(a_{1}+\cdots+a_{m}\right) t} .
$$

Let $D \subset \cap_{k=1}^{m} D_{k}$ be a core for $A$. Then for each $\varphi \in D$ we have

$$
\begin{aligned}
\lim _{t \rightarrow 0} \| & \frac{F(t) \varphi-\varphi}{t}-A \varphi \|_{X} \\
= & \lim _{t \rightarrow 0}\left\|\frac{F_{1}(t) \circ \cdots \circ F_{m}(t) \varphi-\varphi}{t}-A_{1} \varphi-\cdots-A_{m} \varphi\right\|_{X} \\
= & \lim _{t \rightarrow 0} \| F_{1}(t) \circ \cdots \circ F_{m-1}(t) \circ \frac{F_{m}(t) \varphi-\varphi}{t}-A_{m} \varphi \\
& \quad+\frac{F_{1}(t) \circ \cdots \circ F_{m-1}(t) \varphi-\varphi}{t}-A_{1} \varphi-\cdots-A_{m-1} \varphi \|_{X} \\
\leq & \lim _{t \rightarrow 0}\left\|\frac{F_{1}(t) \circ \cdots \circ F_{m-1}(t) \varphi-\varphi}{t}-A_{1} \varphi-\cdots-A_{m-1} \varphi\right\|_{X} \\
\leq & \cdots \leq \lim _{t \rightarrow 0}\left\|\frac{F_{1}(t) \varphi-\varphi}{t}-A_{1} \varphi\right\|_{X} \\
= & 0 .
\end{aligned}
$$

Note, that if some of the $\left(T_{k}(t)\right)_{t \geq 0}$ are known explicitly and if $\left\|T_{k}(t)\right\| \leq e^{a_{k} t}$ for some $a_{k} \geq 0$ then we can take $\bar{F}_{k}(t) \equiv T_{k}(t)$ in the corresponding Feynman formulae.

Example 5.1 (bounded Schrödinger perturbations). Let $X=C_{\infty}\left(\mathbb{R}^{d}\right)$ with the supremum norm, $\left(T_{t}\right)_{t \geq 0}$ be a strongly continuous semigroup with a generator $(A, D(A)),(F(t))_{t \geq 0}$ be Chernoff equivalent to $\left(T_{t}\right)_{t \geq 0}$. Let $V(\cdot): \mathbb{R}^{d} \rightarrow \mathbb{R}$ be a bounded continuous function. Then an operator $A+V$, such that $D(A+V)=D(A)$ and $(A+V) \varphi(q)=A \varphi(q)+V(q) \varphi(q)$ for all $\varphi \in D(A+V)$, generates a strongly continuous semigroup $\left(T_{t}^{A+V}\right)_{t \geq 0}$ on $C_{\infty}\left(\mathbb{R}^{d}\right)$. By Theorem 5.1 the Feynman formula $T_{t}^{A+V}=\lim _{n \rightarrow \infty}\left[e^{\frac{t}{n} V} \circ F(t / n)\right]^{n}$ is valid. In particular, if $\left\|T_{t}\right\| \leq e^{a t}$ for some $a \in[0,+\infty)$ and all $t \geq 0$, then we have $T_{t}^{A+V}=\lim _{n \rightarrow \infty}\left[e^{\frac{t}{n} V} \circ T_{t / n}\right]^{n}$. In both formulae the operator $e^{t V}$ is an operator of multiplication with the function $e^{t V}$.

Example 5.2 (gradient perturbations). Let again $X=C_{\infty}\left(\mathbb{R}^{d}\right)$ with the supremum norm. Let $b(\cdot): \mathbb{R}^{d} \rightarrow \mathbb{R}^{d}$ be a bounded continuous vector field and $\nabla=$ $\left(\frac{\partial}{\partial q_{1}}, \ldots, \frac{\partial}{\partial q_{d}}\right)$. Consider an operator $b \nabla$, such that $b \nabla \varphi(q)=b(q) \nabla \varphi(q)$ for all $\varphi \in D(b \nabla)$. Consider a family $(S(t))_{t \geq 0}$ of operators in $C_{\infty}\left(\mathbb{R}^{d}\right)$ defined by the formula:

$$
S(t) \varphi(q)=\varphi(q+t b(q))
$$


Then $(S(t))_{t \geq 0}$ is Chernoff equivalent to the semigroup $e^{t b \nabla}$ generated by $b \nabla$. Indeed, $S(0)=\mathrm{Id},\|S(t)\|=1$ and for all $\varphi \in D(b \nabla)$

$$
\begin{aligned}
\lim _{t \rightarrow 0}\left\|\frac{S(t) \varphi-\varphi}{t}-b \nabla \varphi\right\|_{\infty} & =\lim _{t \rightarrow 0} \sup _{q \in \mathbb{R}^{d}}\left|\frac{\varphi(q+t b(q))-\varphi(q)}{t}-b(q) \nabla \varphi(q)\right| \\
& \leq \lim _{t \rightarrow 0} t \cdot \sup _{q \in \mathbb{R}^{d}, s \in[0, t]}|b(q) \cdot \operatorname{Hess} \varphi(q+s b(q)) b(q)| \\
& =0,
\end{aligned}
$$

where Hess $\varphi$ is a Hessian of $\varphi$. Hence, $\left.\frac{d}{d t} S(t)\right|_{t=0} \varphi=b \nabla \varphi$ for each $\varphi \in D(b \nabla)$.

Let now $\left(T_{t}\right)_{t \geq 0}$ be a strongly continuous semigroup with a generator $(A, D(A))$ and a family $(F(t))_{t \geq 0}$ be Chernoff equivalent to $\left(T_{t}\right)_{t \geq 0}$. Consider an operator $A+b \nabla$ such that $(A+b \nabla) \varphi(q)=A \varphi(q)+b(q) \nabla \varphi(q)$ for all $\varphi \in D(A+b \nabla)=$ $D(A) \cap D(b \nabla)$. Assume that $A+b \nabla$ generates a strongly continuous semigroup $\left(T_{t}^{A+b \nabla}\right)_{t \geq 0}$ on $C_{\infty}\left(\mathbb{R}^{d}\right)$. Note, that the assumption holds, e.g. if the operator $b \nabla$ is $A$-bounded, i.e. $D(A) \subset D(b \nabla)$ and for all $\varphi \in D(A)$, some $\lambda \in[0,1)$ and $\gamma \geq 0$ the estimate

$$
\|b \nabla \varphi\|_{X} \leq \lambda\|A \varphi\|_{X}+\gamma\|\varphi\|_{X}
$$

holds. In particular, $b \nabla$ is $A$-bounded for $A=-(-\Delta)^{\alpha / 2}, \alpha \in(1,2]$.

By Theorem 5.1 the family $(S(t) \circ F(t))_{t \geq 0}$ is Chernoff equivalent to the semigroup $\left(T_{t}^{A+b \nabla}\right)_{t \geq 0}$ and the Feynman formula $T_{t}^{A+b \nabla}=\lim _{n \rightarrow \infty}[S(t / n) \circ F(t / n)]^{n}$ is valid.

Corollary 5.1 (Hamiltonian Feynman formula for gradient and bounded Schrödinger perturbations of Feller semigroups). Let $b(\cdot), V(\cdot)$ be as in the above examples. Let a function $H(q, p)$ be as in Theorem [3.1, $H(\cdot, D)$ be a $\Psi D O$ with the symbol $H(q, p)$ (see formula (13)) and $F(t)$ be given by the formula (14). We assume that the function $H(q, p)$ satisfies sufficient conditions for $-H(\cdot, D)$ and $A:=-H(\cdot, D)+b \nabla+V$ to be closable and the closures to generate strongly continuous semigroups on $C_{\infty}\left(\mathbb{R}^{d}\right)$. Then by Theorems 3.1, 5.1] and due to the above examples the following Hamiltonian Feynman formula is valid for the semigroup $\left(T_{t}^{A}\right)_{t \geq 0}$, generated by $A=-H(\cdot, D)+b \nabla+V$ :

$$
\begin{aligned}
\left(T_{t}^{A} \varphi\right)\left(q_{0}\right)= & \lim _{n \rightarrow \infty}\left[e^{\frac{t}{n} V} \circ S(t / n) \circ F(t / n)\right]^{n} \varphi\left(q_{0}\right) \\
= & \lim _{n \rightarrow \infty}(2 \pi)^{-d n} \int_{\left(\mathbb{R}^{d}\right)^{2 n}} e^{\frac{t}{n} \sum_{k=1}^{n} V\left(q_{k-1}\right)} e^{i \sum_{k=1}^{n} p_{k} \cdot\left(q_{k-1}-q_{k}+\frac{t}{n} b\left(q_{k-1}\right)\right)} \times \\
& \times e^{-\frac{t}{n} \sum_{k=1}^{n} H\left(q_{k-1}+\frac{t}{n} b\left(q_{k-1}\right), p_{k}\right)} \varphi\left(q_{n}\right) d q_{1} d p_{1} \cdots d q_{n} d p_{n},
\end{aligned}
$$

where again the integrals in the formula must be understood in a proper sense (see Remark 3.2).

Corollary 5.2 (Lagrangian Feynman formula for a mixture of multiplicative and additive perturbations of Feller semigroups). Let $b(\cdot), V(\cdot)$ be as in the above examples, $\widetilde{H}(\cdot, D)$ be as in Section $4, \widetilde{F}(t)$-as in (19). We assume that the function $\widetilde{H}(q, p)$ satisfies sufficient conditions for $-\widetilde{H}(\cdot, D)$ and $B:=-\widetilde{H}(\cdot, D)+b \nabla+V$ to be closable and the closures to generate strongly continuous semigroups on $C_{\infty}\left(\mathbb{R}^{d}\right)$. Then by Theorems 4.1, 5.1 and due to the above examples the following Lagrangian Feynman formula is valid for the semigroup $\left(\widetilde{T}_{t}^{B}\right)_{t \geq 0}$, generated by $B=-\widetilde{H}(\cdot, D)+$ 


$$
\begin{aligned}
b & \nabla+V: \\
& \widetilde{T}_{t}^{B} \varphi\left(q_{0}\right) \\
= & \lim _{n \rightarrow \infty}\left[e^{\frac{t}{n} V} \circ S\left(\frac{t}{n}\right) \circ \widetilde{F}\left(\frac{t}{n}\right)\right]^{n} \varphi\left(q_{0}\right) \\
= & \lim _{n \rightarrow \infty} \int_{\mathbb{R}^{d}} \cdots \int_{\mathbb{R}^{d}} e^{\frac{t}{n} \sum_{k=1}^{n} V\left(q_{k-1}\right)} \varphi\left(q_{n}\right) p_{a\left(q_{0}+b\left(q_{0}\right) \frac{t}{n}\right) \frac{t}{n}}\left(q_{0}+b\left(q_{0}\right) \frac{t}{n}, d q_{1}\right) \times \\
& \times p_{a\left(q_{1}+b\left(q_{1}\right) \frac{t}{n}\right) \frac{t}{n}}\left(q_{1}+b\left(q_{1}\right) \frac{t}{n}, d q_{2}\right) \ldots p_{a\left(q_{n-1}+b\left(q_{n-1}\right) \frac{t}{n}\right) \frac{t}{n}}\left(q_{n-1}+b\left(q_{n-1}\right) \frac{t}{n}, d q_{n}\right) .
\end{aligned}
$$

Example 5.3 (Lagrangian Feynman formula for perturbations of the heat semigroup). Let again $b(\cdot), V(\cdot)$ be as before. Consider $A=\frac{1}{2} \Delta ; A$ is the generator of the heat semigroup $\left(T_{t}\right)_{t \geq 0}: T_{t} \varphi(q)=(2 \pi t)^{(-d / 2)} \int_{\mathbb{R}^{d}} e^{-\frac{|q-y|^{2}}{2 t}} \varphi(y) d y$. Then by Theorem [5.1 the Lagrangian Feynman formula is valid (cf. [7], [8]) for the semigroup $\left(T_{t}^{C}\right)_{t \geq 0}$ generated by $C:=\frac{1}{2} \Delta+b \nabla+V$ :

$$
\begin{gathered}
T_{t}^{C} \varphi\left(q_{0}\right)=\lim _{n \rightarrow \infty}(2 \pi t / n)^{(-d n / 2)} \int_{\mathbb{R}^{d}} \cdots \int_{\mathbb{R}^{d}} e^{\frac{t}{n} \sum_{k=1}^{n} V\left(q_{k-1}\right)} e^{-\sum_{k=1}^{n} \frac{\left|q_{k-1}+b\left(q_{k-1}\right) t / n-q_{k}\right|^{2}}{2 t / n}} \times \\
\times \varphi\left(q_{n}\right) d q_{1} \cdots d q_{n} .
\end{gathered}
$$

Since $|x+b(x) t-y|^{2}=|x-y|^{2}+2 t b(x)(x-y)+t^{2}|b(x)|^{2}$ then

$$
\begin{aligned}
T_{t}^{b \nabla+V} & \varphi\left(q_{0}\right)=\lim _{n \rightarrow \infty} \int_{\mathbb{R}^{d}} \cdots \int_{\mathbb{R}^{d}} e^{\frac{t}{n} \sum_{k=1}^{n} V\left(q_{k-1}\right)} e^{-\sum_{k=1}^{n} b\left(q_{k-1}\right)\left(q_{k-1}-q_{k}\right)} \times \\
& \times e^{-\frac{t}{2 n} \sum_{k=1}^{n}\left|b\left(q_{k-1}\right)\right|^{2}} p_{t / n}^{B M}\left(q_{0}-q_{1}\right) \cdots p_{t / n}^{B M}\left(q_{n-1}-q_{n}\right) \varphi\left(q_{n}\right) d q_{1} \cdots d q_{n},
\end{aligned}
$$

where $p_{t}^{B M}(x)=(2 \pi t)^{(-d / 2)} \exp \left\{-\frac{|x|^{2}}{2 t}\right\}$ is the transition density of Brownian motion. Therefore, one can show, that the limit in the right hand side of the last formula coincides with the functional integral

$$
\mathbb{E}^{q_{0}}\left[e^{\int_{0}^{t} V\left(\xi_{\tau}\right) d \tau} e^{\int_{0}^{t} b\left(\xi_{\tau}\right) d \xi_{\tau}} e^{-\frac{1}{2} \int_{0}^{t}\left|b\left(\xi_{\tau}\right)\right|^{2} d \tau} f\left(\xi_{t}\right)\right]
$$

with respect to Wiener measure concentrated on the paths starting at $q_{0}$. Hence, the machinery of Feynman formulae provides not only another way to prove the Feynman-Kac formula (21) and Girsanov's formula but also to extend them for the case of variable diffusion coefficients (cf. [8]).

\section{ACKNOWLEDGEMENTS}

Financial support by the Deutsche Forschungsgemeinschaft through the project SCHI 419/7-1, by the Russian Foundation for Basic Research through the project 10-01-00724-a, by the grant of the President of Russian Federation through the projects MK-943.2010.1 and MK-4255.2012.1, by the Erasmus Mundus Action 2 Programme of the European Union is gratefully acknowledged.

\section{REFERENCES}

[1] Berg, C., Forst G., Potential Theory on Locally Compact Abelian Groups, Springer, Ergeb. Math. Grenzgeb. Bd. 87, Berlin 1975.

[2] Bony, J.-M., Courrège, Ph., Priouret P., Semi-groupes de Feller sur une variété à bord compacte et problème aux limites intégro- différentiels du second ordre donnant lieu au principe du maximum, Ann. Inst. Fourier 18.2 (1968), 369-521.

[3] B. Böttcher, Ya.A. Butko, R.L. Schilling and O.G. Smolyanov. Feynman formulae and path integrals for some evolutionary semigroups related to $\tau$-quantization, Rus. J. Math. Phys., 18 N 4 (2011), 387-399. 
[4] Böttcher B., Schilling R.L., Approximation of Feller processes by Markov chains with Lévy increments. Stochastics and Dynamics 9 (2009), 71-80.

[5] Butko Ya. A., Feynman formulas and functional integrals for diffusion with drift in a domain on a manifold. Math. Notes. 2008. V. 83. N. 3-4. P. 301-316.

[6] Butko Ya. A., Function integrals corresponding to a solution of the Cauchy-Dirichlet problem for the heat equation in a domain of a Riemannian manifold, J. of Math. Sci., 2008. V.151. N. 1. P. 2629-2638.

[7] Butko Ya., Grothaus M., Smolyanov O.G., Feynman Formula for a Class of Second-Order Parabolic Equations in a Bounded Domain. Doklady Math. 2008. V. 78. N. 1. P. 590-595.

[8] Butko Ya., Grothaus M., Smolyanov O.G., Lagrangian Feynman Formulae for Second Order Parabolic Equations in Bounded and Unbounded Domains. IDAQP 2010. V. 13. N. 3. P. 377-392.

[9] Butko Ya., Schilling R.L., Smolyanov O.G., Feynman formulae for Feller semigroups, Doklady Math., 2010. V.82. N.2. P. 679-683, DOI 10.1134/S1064562410050017.

[10] Ya.A. Butko, R.L. Schilling and O.G. Smolyanov. Hamiltonian Feynman-Kac and Feynman formulae for dynamics of particles with position-dependent mass. Int. J. Theor. Phys. (2010), 10 p., DOI 10.1007/s10773-010-0538-4.

[11] Carmona R., Masters W.Ch., Simon B., Relativistic Schroedinger Operators: Asymptotic Behavior of the Eigenfunctions. J. Func. Anal. 1990.V.91. P. 117-142.

[12] Chernoff P., Product formulas, nonlinear semigroups and addition of unbounded operators. Mem. Am. Math. Soc., 140 (1974).

[13] Courrège, Ph., Sur la forme intégro-différentielle des opérateurs de $C_{K}^{\infty}$ dans $C$ satisfaisant au principe du maximum, Séminaire de Théorie du Potentiel (1965/66) exposé 2, 38 pp.

[14] Dorroh J.R., Contraction semi-groups in a function space. Pacific J.Math. 19 N1 (1966), 35-38.

[15] Ethier, S.E., Kurtz T.G., Markov Processes: Characterization and Convergence, Wiley, Ser. Probab. Math. Stat., New York 1986.

[16] Feynman R. P., Space-time approach to nonrelativistic quantum mechanics. Rev. Mod. Phys. 20 (1948), 367-387.

[17] Feynman R.P., An Operator Calculus Having Applications in Quantum Electrodynamics, Phys. Rev., 84 (1951), 108-128.

[18] Gadella M., Smolyanov O.G., Feynman Formulas for Particles with Position-Dependent Mass. Doklady Math. 2007. V. 77. N. 1. P. 120-123.

[19] Hwang I.L., The $L_{2}$-boundness of pseudodifferential operators. Trans. A.M.S. 302 N 1 (1987), 55-76.

[20] Ichinose T., Tamura H., Imaginary-time integral for a relativistic spinless particle in an electromagnetic field. Commun. Math. Phys., 105: 239-257, 1986.

[21] Jacob N., Characteristic functions and symbols in the theory of Feller processes. Potential Anal. 8 (1998), 61-68.

[22] Jacob N., Pseudo-differential operators and Markov processes. Vol. 1-3. Imperial College Press, 2001.

[23] Jacob N., Potrykus A., Roth's Method Applied to Some Pseudo-Differential Operators with Bounded Symbols. A Case Study. Rendiconti del Circ. Mat. di Palermo. 2005. Ser. II. N. 76. P. 45-57.

[24] Jacob N., Schilling R.L., Lévy-type processes and pseudo differential operators, O. BarndorffNielsen, T. Mikosch and S. Resnick (eds.): Lévy processes: theory and applications, Birkhäuser, Boston 2001, 139-167.

[25] Nelson E., Feynman integrals and the Schrödinger equation. J. Math. Phys. 3 (1964), 332343.

[26] Obrezkov O.O., The Proof of the Feynman-Kac Formula for Heat Equation on a Compact Riemannian Manifold, IDAQP, 2003. V. 6. N. 2. P. 311-320.

[27] Obrezkov O., Smolyanov O.G., Truman A., The Generalized Chernoff Theorem and Randomized Feynman Formula. Doklady Math. 2005. V. 71. N. 1. P. 105-110.

[28] Reed M., Simon B., Methods of Modern Mathematical Physics, Vol. I, Academic Press, 1980.

[29] Sato K., Lévy Processes and Infinitely Divisible Distributions. Cambridge Univ. Press. 1999.

[30] Schilling R.L., Conservativeness of semigroups generated by pseudo differential operators, Potential Anal. 9 (1998), 91 - 104.

[31] Schilling R.L. and Schnurr A., The symbol associated with the solution of a stochastic differential equation. El. J. Probab. 15 (2010), 1369-1393.

[32] Smolyanov O.G. and Shamarov N.N., Feynman and Feynman-Kac formulae for evolutionary equations with Vladimirov operator, Doklady Math. 77 (2008), 345-349.

[33] Smolyanov O.G., Tokarev A.G., Truman A., Hamiltonian Feynman path integrals via the Chernoff formula. J. Math. Phys. 2002. V.43. N. 10. P. 5161-5171. 
[34] Smolyanov O.G., Weizsäcker H.v. and Wittich O., Diffusion on compact Riemannian manifolds, and surface measures. Doklady Math. 61 (2000), 230-234.

[35] Smolyanov O.G., Weizsäcker H. v., Wittich O., Brownian Motion on a Manifold as Limit of Stepwise Conditioned Standard Brownian Motions. In: Stochastic Proceses, Physics and Geometry: New Interplays. II: A Volume in Honor of Sergio Albeverio. Ser. Conference Proceedings. Canadian Math. Society. Providence: AMS. 2000. V. 29. P. 589-602.

[36] Smolyanov O.G., Weizsäcker H.v., Wittich O., Surface Measures and Initial Boundary Value Problems Generated by Diffusions with Drift. Doklady Math. 2007. Vol. 76. N. 1. P. 606-610.

[37] Smolyanov O.G., Weizsäcker H. v., Wittich O., Chernoff's Theorem and Discrete Time Approximations of Brownian Motion on Manifolds. Potent. Anal. 2007. V. 26. N. 1. P. 1-29.

[38] Telyatnikov I.V., Smolyanov-Weizsäcker surface measures generated by diffusions on the set of trajectories in Riemannian manifolds, IDAQP. 2008. V. 11. N. 1. P. 21-31.

[39] von Waldenfels, W., Eine Klasse stationärer Markowprozesse, Berichte der Kernforschungsanlage Jülich (1961).

[40] von Waldenfels, W., Positive Halbgruppen auf einem n-dimensionalen Torus, Arch. Math. 15 (1964), 191-203.

Department of Fundamental Sciences, Bauman Moscow State Technical University, 105005, 2nd Baumanskaya str., 5, Moscow, Russia, yanabutko@yandex.ru

Institut für Mathematische Stochastik, Technische Universität Dresden, Zellescher Weg, 12-14, D-01069 Dresden, Germany, REne.SChilling@tu-Dresden.DE

Department of Mechanics and Mathematics, Lomonosov Moscow State University, 119992, Vorob'evy Gory, 1, Moscow, Russia, Smolyanov@yandex.ru 